\title{
Simultaneous Obstacle Avoidance and Target Tracking of Multiple Wheeled Mobile Robots With Certified Safety
}

\author{
Xiaoxiao $\mathrm{Li}^{(}{ }^{(}$, Zhihao $\mathrm{Xu}^{(}$, Member, IEEE, Shuai Li, Senior Member, IEEE, Zerong Su, \\ and Xuefeng Zhou ${ }^{\circledR}$, Member, IEEE
}

\begin{abstract}
Collision avoidance plays a major part in the control of the wheeled mobile robot (WMR). Most existing collision-avoidance methods mainly focus on a single WMR and environmental obstacles. There are few products that cast light on the collision-avoidance between multiple WMRs (MWMRs). In this article, the problem of simultaneous collision-avoidance and target tracking is investigated for MWMRs working in the shared environment from the perspective of optimization. The collisionavoidance strategy is formulated as an inequality constraint, which has proven to be collision free between the MWMRs. The designed MWMRs control scheme integrates path following, collision-avoidance, and WMR velocity compliance, in which the path following task is chosen as the secondary task, and collisionavoidance is the primary task so that safety can be guaranteed in advance. A Lagrangian-based dynamic controller is constructed for the dominating behavior of the MWMRs. Combining theoretical analyses and experiments, the feasibility of the designed control scheme for the MWMRs is substantiated. Experimental results show that if obstacles do not threaten the safety of the WMR, the top priority in the control task is the target track task. All robots move along the desired trajectory. Once the collision criterion is satisfied, the collision-avoidance mechanism is activated and prominent in the controller. Under the proposed scheme, all robots achieve the target tracking on the premise of being collision free.
\end{abstract}

Index Terms-Collision avoidance, motion planning, multiple wheeled mobile robots (MWMRs), quadratic programming.

Manuscript received January 8, 2021; accepted March 26, 2021. This work was supported in part by the Guangdong Province Key Areas Research and Development Program under Grant 2019B090919002 and Grant 2020B090925001; in part by the National Natural Science Foundation of China under Grant 62003102; in part by the Natural Science Foundation of Guangdong Province under Grant 2020A1515010631; in part by the Foshan Key Technology Research Project under Grant 1920001001148; in part by the Foshan Innovation and Entrepreneurship Team Project under Grant 2018IT100173; and in part by the GDAS' Project of Thousand Doctors (Postdoctors) Introduction under Grant 2020GDASYL-20200103128. This article was recommended by Associate Editor S. Nahavandi. (Corresponding author: Xuefeng Zhou.)

Xiaoxiao Li, Zhihao $\mathrm{Xu}$, Zerong $\mathrm{Su}$, and Xuefeng Zhou are with the Guangdong Key Laboratory of Modern Control Technology, Institute of Intelligent Manufacturing, Guangdong Academy of Sciences, Guangzhou 510070, China (e-mail: xiaoxiaoli1993@sina.com; zh.xu@giim.ac.cn; zr.su@giim.ac.cn; xf.zhou@giim.ac.cn).

Shuai Li is with the School of Engineering, Swansea University, Swansea SA2 8PP, U.K., and also with the Technical Development Department, Foshan Tri-Co Intelligent Robot Technology Company Ltd., Foshan 528315, China (e-mail: shuaili@ieee.org).

Color versions of one or more figures in this article are available at https://doi.org/10.1109/TCYB.2021.3070385.

Digital Object Identifier 10.1109/TCYB.2021.3070385

\section{INTRODUCTION}

$\mathbf{G}$ ENERALLY speaking, any mechanical device equipped with a wheeled mobile driving mechanism can be viewed as a wheeled mobile robot (WMR). The WMR has wider covering and social applications as well as strong practicability. With the gradual maturation of several intelligent technologies, including artificial intelligence, computer vision, and the Internet of Things, brilliant advances in controlling WMRs have been made, including the Google Driverless Car, freight logistics robots such as Kiva, and agricultural mobile robots such as BoniRob. Especially, in recent years, a cooperative vehicle-infrastructure system, which is a critical development direction of intelligent transportation systems and intelligent logistics among others, has been paid more attention, making the research of the WMR in full swing. Many achievements ranging from a single WMR to multiple WMRs (MWMRs) have been reported, such as in object/obstacle detection and identification [1]-[3]; robotenvironmental/human interaction enhancement [4], [5]; and localization/navigation [6]-[8], which opens a convenient window for the path planning and following [2], [9]-[11] of WMRs; consensus tracking [12], [13]; etc.

With the increasing number of WMRs in the urban environment and the high safety accident risk, an increasing number of scholars and industrial people have focused on the safety assurance. This is mainly because many uncertain factors and interference, such as pedestrians, buildings, and other team members, may exist during the desired behavior execution. On balance, the obstacle avoidance method for WMRs can be divided into two classes: 1) global and 2) local methods. The well-known $A^{*}$ and $D^{*}$, and rapidly-exploring random tree (RRT) algorithms are global planning methods. These methods usually need global information of the current environment; positions of the target and obstacles must be known and accurate. Typical local planning methods include artificial potential field (APF)-based algorithms [14], [15]; metaheuristic-based algorithms [16]; artificial neural-network (ANN)-based algorithms [14], [17]; fuzzy-logic (FL)-based algorithms [18]; etc. This kind of algorithm only needs local information about the surrounding environment and does not need to understand the global situation, which is considered to be promising.

Algorithms differ in how they avoid obstacles and have their respective advantages and disadvantages. For the APF-based 
method, the robot is assumed to move in a virtual potential field consisting of an attractive potential field and a repulsive potential field. The target is described as an attractive force, and the obstacle is described as a repulsive force. The resultant force is used to decide the next direction of the robot. The method is easy to understand because the mathematical concept, together with the code implementation, is simple. However, different potential functions are required for different scenes to avoid a local optimum or the problem that the target is nonreachable. A metaheuristic-based method is able to generate the shortest path from the initial position to the target position. However, it requires large memory compared to the ANN-based method. In terms of path generation, metaheuristic-based methods take time, and a sized population is needed to obtain the desired result. ANN-based methods can learn and can model linear, nonlinear, or complex relationships. Compared to the GA-based method, the ANN method will fail in a highly chaotic environment [19]. Furthermore, a great quantity of data is needed for training, so training time is long. Inspired by human reasoning, FL-based methods can handle uncertainties and imprecise information using linguistic rules and can make inferences using environmental data [18]. However, a fuzzy rule base needs to be constructed and tuned by a human expert. It is difficult to maintain the correctness, consistency, and completeness of a fuzzy rule base.

To achieve better obstacle avoidance behavior, the fusion of multiple types of obstacle avoidance methods is becoming a promising method. To avoid dynamic obstacles, Li et al. [14] incorporated the velocity of obstacles relative to the robots into the potential function. An ANN trained by previous positions of the obstacle was used to predict the position and velocity of the obstacle. In [20], a neural network and fuzzy control were together used for static obstacle avoidance of a single WMR. In [15], an artificial-potential-field-resistance-networkbased trajectory tracking method for autonomous vehicles was proposed. By assigning a potential function, the collisionfree requirement was achieved. In [21], aiming at finding an optimal path for a WMR from source to destination across multiple obstacles, a genetic-learning-based five-level neuralnetwork fuzzy approach was proposed, where the obstacle was denoted by its bounding rectangle. In addition, a pulsecoupled neural-network method is proposed in [17] to generate a collision-free path for a mobile robot under a dynamic environment. The method requires no prior knowledge of the target or obstacles and can give the shortest path from the source to the target. However, one weakness is that global knowledge of the current environment is assumed to be available, which is not realistic in real applications. In [22], two fuzzy controllers were designed to independently dominate a WMR to escape the encountered environmental obstacles and track the desired trajectory. Based on the interval type-2 fuzzy neural network (IT2FNN), the collision-free behavior was achieved in [23]. The above-mentioned works only consider a single WMR. For the multirobot scene, in [24] and [25], the leader-followerbased formation control problem of a group of mobile robots was considered. In [24], other robots in a shared environment were treated as obstacles. By applying a robust integral of the sign of the error (RISE) method, it was ensured that the entire formation was asymptotically stable during the obstacle avoidance. In [25], a multiregion obstacle avoidance method was proposed. Robots performed different operations based on the divided region criteria. However, robots would stop when there were obstacles in front of them before making a decision.

Recently, addressing the collision-avoidance problem from the perspective of optimization has become popular. A typical feature of the method is that the collision-free mechanism is described as an inequality constraint [26]-[29]. At first, this method was mainly used to address the redundant resolution problem of the manipulator [30]-[36]. The basic idea is that the redundant resolution problem is formulated as a quadratic programming minimization scheme, and it is solved from the perspective of optimization. Using this method, multiple objectives, such as robot joint constraints, target tracking, and repetitive motion planning can be simultaneously achieved by describing them as attachment equality or inequality constraints. Apart from being applied to manipulators, the inequality collision-avoidance method was used for a wheeled mobile manipulator and dual redundant robot manipulators in [37] and [38], respectively. Compared to other collision-avoidance methods, the method is simple and easy to implement. By adaptively calculating the distance between the robot and the obstacle, whose position is transmitted by a camera, the command that tracks or avoids obstacles is made based on the setting safety threshold. Motivated by this, we extend the inequality-based collision-avoidance method to MWMRs and propose a simultaneous obstacle avoidance and trajectory tracking scheme for MWMRs working in a shared environment. Although this collision-avoidance method has been applied to manipulators and mobile manipulators, it has seldom been applied to MWMRs. Even for the robot manipulator, there is little consideration of multimanipulator collision-avoidance. To highlight our work, a comparison between our work and the above-mentioned work is listed in Table I. Moreover, the main contributions of this study are summarized as follows.

1) Simultaneous collision-avoidance and target tracking are investigated for MWMRs working in a shared environment. Not only considering the environmental obstacle, we also cast light on collision-avoidance between MWMRs.

2) A multiobjective simultaneous optimization scheme integrating target tracking, collision-avoidance, and robot velocity compliance is synthesized, and a Lagrangian-based controller is designed for solving it. Theoretical analyses and experiments substantiate the effectiveness of the constructed controller.

3) This is the first work to extend the inequality-based collision-avoidance method to MWMRs. Although this collision-avoidance method has been applied to manipulators and mobile manipulators, it has seldom been applied to MWMRs. Even for manipulators, there is also little consideration of multimanipulator collisionavoidance. 
TABLE I

COMPARISON BETWEEN THE CURRENT STUDY AND EXISTING WORK

\begin{tabular}{clcccc}
\hline Literature & \multicolumn{1}{c}{ Methods } & $\begin{array}{c}\text { Physical } \\
\text { constraint }\end{array}$ & $\begin{array}{c}\text { Environmental } \\
\text { obstacles }\end{array}$ & $\begin{array}{c}\text { Collision avoidance } \\
\text { between robots }\end{array}$ & Tasks \\
\hline$[14]$ & APF-ANN-integrated & No & Dynamic & No & Point-to-point navigation and obstacle avoidance \\
{$[20]$} & Neuro-fuzzy-integrated & No & Static & No & Obstacle avoidance \\
{$[21]$} & Genetic-neuro-fuzzy-integrated & No & Static & No & Point-to-point navigation and obstacle avoidance \\
{$[22]$} & FL-based & No & Static & No & Point-to-point navigation and obstacle avoidance \\
{$[23]$} & IT2FNN-based Reactive method & No & Static & No & Obstacle avoidance and position stabilization \\
{$[37]$} & Optimization & Yes & Static & No & Simultaneous obstacle avoidance and target tracking \\
{$[24]$} & NN-RISE-integrated & No & Static and dynamic & Yes & Simultaneous obstacle avoidance and formation \\
{$[25]$} & Steering-based region-division method & No & Static and dynamic & Yes & Simultaneous obstacle avoidance and formation \\
{$[38]$} & Optimization & Yes & Static & Yes* & Simultaneous obstacle avoidance and target tracking \\
This paper & Optimizaiton & Yes & Static and dynamic & Yes & Simultaneous obstacle avoidance and target tracking \\
\hline
\end{tabular}

* In this study, the employed robot is a dual manipulator robot.

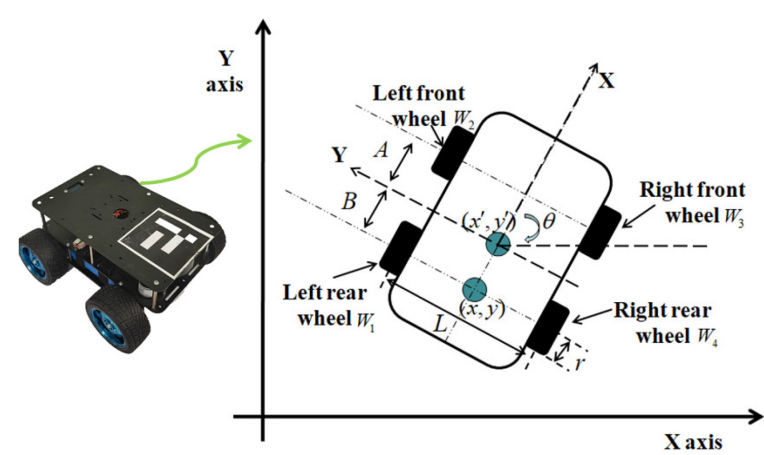

Fig. 1. Model of the WMR used in this article.

\section{Kinematic Model, Obstacle Avoidance, And PROBLEM STATEMENT}

In this part, a kinematic model of a WMR with four differentially driven wheels is first given to lay a basis. We then introduce the collision-avoidance strategy for the WMR, and the scheme is further formulated as an inequality. Finally, based on it, a multiobjective optimization strategy simultaneously integrating collision-avoidance and trajectory tracking is given for MWMRs in a fashion of optimization.

\section{A. Kinematic Model of WMR}

A schematic diagram of the employed mobile robot in the current study is shown on the left-hand side of Fig. 1. The right-hand side is the kinematics schematic of the mobile robot. The following assumptions are considered in the robot's skid steer motion [39], [40].

1) The mass center of the robot is located at the geometric center of the body frame.

2) The mobile robot is symmetrical.

3) The two wheels of each side rotate at the same speed.

4) The robot is running on a firm ground surface and four wheels are always in contact with the ground surface.

5) The robot is subject to a Pfaffian constraint, that is, the contact between four wheels and the ground surface is pure rolling and nonslipping.
Assume that point $Z$ is the posture of the WMR at time $t$, which is defined by the robot's planner coordinates $(x, y)$ in the local frame and its heading angle $\theta$. Based on the above assumptions, the kinematic model of the WMR with four fixed standard wheels that are differentially driven can be described as

$$
\frac{d Z}{d t}=\mathrm{BW}
$$

in which

$$
\begin{aligned}
& Z=\left[\begin{array}{l}
x \\
y \\
\theta
\end{array}\right] \in \mathbb{R}^{3 \times 1}, \quad B=\left[\begin{array}{cc}
\cos \theta & 0 \\
\sin \theta & 0 \\
0 & 1
\end{array}\right] \in \mathbb{R}^{3 \times 2} \\
& W=\left[\begin{array}{c}
v \\
w
\end{array}\right]=\left[\begin{array}{c}
\frac{r}{2}\left(u_{r}+u_{l}\right) \\
\frac{r}{L}\left(u_{r}-u_{l}\right)
\end{array}\right] \in \mathbb{R}^{2 \times 1} .
\end{aligned}
$$

$v$ and $w$ denote the linear and angular velocity of a WMR, respectively, with $v=(r / 2)\left(u_{r}+u_{l}\right)$ and $w=(r / L)\left(u_{r}-u_{l}\right)$. $u_{l}$ and $u_{r}$ are the velocities of the left wheel and the right wheel of the WMR, respectively. Parameter $r$ denotes the radius of the driven wheel. $L$ is the lateral wheel bases. Therefore, (1) can be further written as

$$
\left[\begin{array}{c}
\dot{x} \\
\dot{y} \\
\dot{\theta}
\end{array}\right]=\left[\begin{array}{c}
\frac{r}{2}\left(u_{r}+u_{l}\right) \cos \theta \\
\frac{r}{2}\left(u_{r}+u_{l}\right) \sin \theta \\
\frac{r}{L}\left(u_{r}-u_{l}\right)
\end{array}\right] \in \mathbb{R}^{3 \times 1} .
$$

Further details of the kinematics analysis of the WMR can be found in [39]. We define $u=\left[u_{l}, u_{r}\right]^{\mathrm{T}} \in \mathbb{R}^{2 \times 1}$, where $\mathrm{T}$ denotes the transpose of the matrix. Obviously, the action vector $u=\left[u_{l}, u_{r}\right]^{\mathrm{T}}$ directly affects the next motion of the robot. To recover point control, we assume that $Z_{\text {ref }}$ is a reference point of a WMR with the definition of $Z_{\text {ref }}=$ $\left[x+d_{0} \cos \theta, y+d_{0} \sin \theta\right]^{\mathrm{T}}$, where $0<d_{0}<L$. The reason for this is that the position $(x, y)$ and the heading angle $\theta$ cannot be simultaneously stabilized by a time-invariant feedback [41]. When $d_{0}=B$, the mass center of the robot will be chosen to be the reference point. Computing the time derivative of $Z_{\text {ref }}$, combined with (4), we can obtain

$$
\dot{Z}_{\text {ref }}=A u \in \mathbb{R}^{2 \times 1}
$$




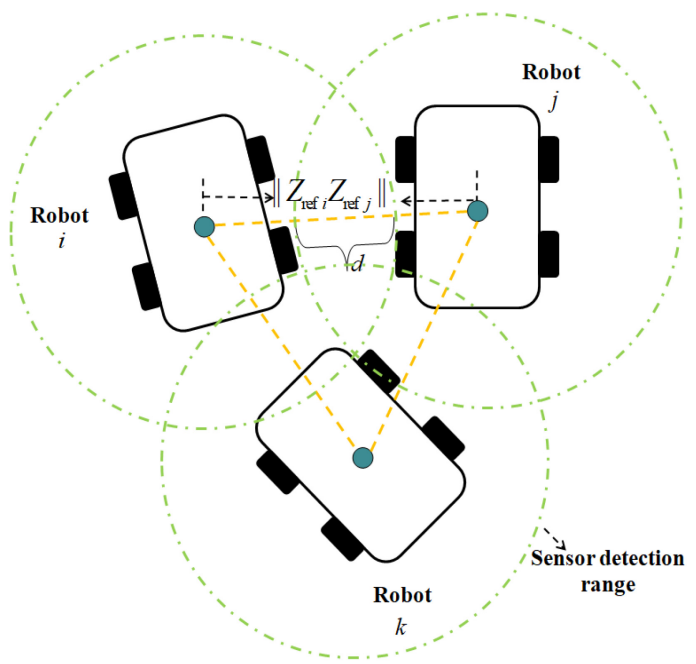

Fig. 2. Basic description of the collision-free strategy proposed in this article. When a WMR $j$ enters the sensor range of a WMR $i$, if the collision criterion $\left\|Z_{\text {ref }_{i}} Z_{\text {ref }_{j}}\right\|<d$ is satisfied, the obstacle avoidance strategy will assume control, enabling the robots $i$ and $j$ to always keep a safe distance that is greater than or equal to $d$.

with

$$
A=\left[\begin{array}{ll}
\frac{r \cos \theta}{2}+\frac{r d_{0} \sin \theta}{L} & \frac{r \cos \theta}{2}-\frac{r d_{0} \sin \theta}{L} \\
\frac{r \sin \theta}{2}-\frac{r d_{0} \cos \theta}{L} & \frac{r \sin \theta}{2}+\frac{r d_{0} \cos \theta}{L}
\end{array}\right] \in \mathbb{R}^{2 \times 2}
$$

\section{B. Collision-Avoidance Strategy}

In principle, if no collision between WMR $i$ and WMR $j$ happens, the distance between their reference points should always be kept outside a safe range, that is, $\left\|Z_{\mathrm{ref}_{i}} Z_{\mathrm{ref}_{j}}\right\| \geq$ $d$, where $\left\|Z_{\mathrm{ref}_{i}} Z_{\mathrm{ref}_{j}}\right\|=\sqrt{\left(Z_{\mathrm{ref}_{i}}-Z_{\mathrm{ref}_{j}}\right)^{\mathrm{T}}\left(Z_{\mathrm{ref}_{i}}-Z_{\mathrm{ref}_{j}}\right)}$ is the Euclidean norm of $Z_{\mathrm{ref}_{i}} Z_{\mathrm{ref}_{j}}$. The safe distance $d$ is a positive constant determined by the user. $Z_{\mathrm{ref}_{i}}$ and $Z_{\mathrm{ref}_{j}}$ are the reference points of WMRs $i$ and $j$, respectively, where $i=1, \ldots, N$, $j=1, \ldots, N$, with $i \neq j . N$ is the number of WMRs. Fig. 2 gives a schematic of the elaborated collision-free strategy. By enabling the distance between the WMRs $j$ and $i$ to always keep a safe distance that is greater than or equal to $d$, the collision-free strategy is achieved.

In order to ensure that $\left\|Z_{\mathrm{ref}_{i}} Z_{\mathrm{ref}_{j}}\right\| \geq d$, a common operation is

$$
\partial\left(\left\|Z_{\text {ref }_{i}} Z_{\text {ref }_{j}}\right\|-d\right) / \partial t \geq-k_{1}\left(\left\|Z_{\text {ref }_{i}} Z_{\text {ref }_{j}}\right\|-d\right)
$$

with $k_{1}>0$, which is a control gain parameter [26]-[28]. In order to remove the radical sign, we define $\left\|Z_{\text {ref }_{i}} Z_{\text {ref }_{j}}\right\|^{2} \geq d^{2}$. Similar to (7), therefore, we have

$$
2\left(Z_{\mathrm{ref}_{i}}-Z_{\mathrm{ref}_{j}}\right)^{\mathrm{T}}\left(\dot{Z}_{\mathrm{ref}_{i}}-\dot{Z}_{\mathrm{ref}_{j}}\right) \geq-k_{1} \hat{e}
$$

where $\hat{e}=\left\|Z_{\mathrm{ref}_{i}} Z_{\mathrm{ref}_{j}}\right\|^{2}-d^{2}$. Combined with (5), (8) can be further rewritten as

$$
2\left(Z_{\mathrm{ref}_{i}}-Z_{\mathrm{ref}_{j}}\right)^{\mathrm{T}}\left(A_{i} u_{i}-A_{j} u_{j}\right) \geq-k_{1} \hat{e} .
$$

The above is for a safety guarantee strategy among the robots. For collision-avoidance between a WMR $i$ and an environmental obstacle $O_{j}$ (it may be static or dynamic), we establish the following equation:

$$
2\left(Z_{\text {ref }_{i}}-O_{j}\right)^{\mathrm{T}}\left(A_{i} u_{i}\right) \geq-k_{1}\left(\left\|Z_{\text {ref }_{i}} O_{j}\right\|^{2}-d^{2}\right)
$$

where $O_{j}$ denotes the Cartesian coordinates of the detected environmental obstacle $j$.

Remark 1: Based on (5) and (6), since the WMR parameters $r$ and $L$ are fixed and known, $A$ is uniquely determined by $\theta$, which is related to $u_{l}$ and $u_{r}$. Therefore, $A$ can be measured in real time based on the feedback of $u_{l}$ and $u_{r}$. Moreover, the WMR senses environmental information surrounding itself with a sensor. The positions of both the static and dynamic environmental obstacles are easy to obtain in real time through a camera, which has been achieved in [1]-[3], [6], and [7]. Camera calibration errors were also solved [42], [43]. Consequently, the collision-avoidance strategy introduced in this article is feasible in real implementations. Notice that this study focuses on collision-avoidance; object/obstacle detection and identification are not within the investigation scope of this study. In this article, positions of obstacles are assumed to be known in advance.

\section{Unified Optimization Problem Statement}

In this article, we aim at achieving three objectives. For Objective 1 (the desired trajectory tracking task), we assume that $Z_{\mathrm{cmd}_{i}}$ is the desired trajectory point coordinate that the WMR $i$ is expected to follow. To achieve $Z_{\mathrm{ref}_{i}} \rightarrow Z_{\mathrm{cmd}_{i}}$, we define

$$
\dot{Z}_{\mathrm{ref}_{i}}=\dot{Z}_{\mathrm{cmd}_{i}}-k_{2}\left(Z_{\mathrm{ref}_{i}}-Z_{\mathrm{cmd}_{i}}\right)
$$

which will be shown to enable the desired trajectory tracking error $\ell=\left\|Z_{\mathrm{ref}_{i}}-Z_{\mathrm{cmd}_{i}}\right\|$ to globally converge to 0 in the ensuing section.

Objective 2 (the WMR velocity limit)

$$
u_{i}^{-} \leq u_{i} \leq u_{i}^{+}
$$

where $u_{i}^{+}$and $u_{i}^{-}$are the upper and lower bounds of the wheel velocity of WMR $i$, respectively.

Objective 3 (collision-avoidance) was introduced in the previous section. In order to achieve these three objectives at the same time, we describe them as a unified minimization optimization problem, where the trajectory tracking task is constructed as the cost function, and the velocity limit and collision-avoidance are attached as two inequality constraints. Specifically, for the WMR $i$, we have

$$
\begin{aligned}
\min _{u} & \left\|\dot{Z}_{\mathrm{ref}_{i}}-\dot{Z}_{\mathrm{cmd}_{i}}+k_{2}\left(Z_{\mathrm{ref}_{i}}-Z_{\mathrm{cmd}_{i}}\right)\right\|^{2} \\
\text { s.t. } & u_{i}^{-} \leq u_{i} \leq u_{i}^{+} \\
& 2\left(Z_{\mathrm{ref}_{i}}-Z_{\mathrm{ref}_{j}}\right)^{\mathrm{T}}\left(A_{i} u_{i}-A_{j} u_{j}\right) \geq-k_{1} \hat{e} .
\end{aligned}
$$

Note that due to similarity, we only give the inequality constraint, representing the multiple MWRs' collision-avoidance. In the next part, we construct a dynamic Lagrangian-based controller for solving the resultant (13). Under its control, outside the nonsafety region $\left(\left\|Z_{\mathrm{ref}_{i}} Z_{\text {ref }_{j}}\right\| \geq d\right)$, the mobile robot tracks its desired trajectory with a rational velocity satisfying (13b). If the collision criterion is satisfied, that is, 
$\left\|Z_{\mathrm{ref}_{i}} Z_{\mathrm{ref}_{j}}\right\|<d$, the collision-avoidance mechanism assumes control, enabling all WMRs to keep a safe distance $\geq d$ from their surrounding WMRs.

\section{LAGRANGIAN-BASED CONTROLLER DESIGN}

In this part, a Lagrangian-based controller will be designed to find a minimal solution to the resultant optimization problem (13). The optimization method employed by the developed controller is the classical Lagrange multiplier method. Through a suitable choice of controller parameters, the controller generates a linear velocity $v$ and an angular velocity $w$ for each WMR to drive them along their respective desired trajectories as much as possible while ensuring safety. Before designing the controller, for simplicity, we first give the combined-formed optimization problem formulation considering $N$ robots and then introduce a specified-designed Lagrangian-based controller.

\section{A. Optimization Problem Reformulation}

For (13c), it can be rewritten as

$$
-2\left(Z_{\mathrm{ref} i}-Z_{\mathrm{ref} j}\right)^{\mathrm{T}}\left[\begin{array}{ll}
I_{2} & -I_{2}
\end{array}\right]\left[\begin{array}{cc}
A_{i} & 0 \\
0 & A_{j}
\end{array}\right]\left[\begin{array}{l}
u_{i} \\
u_{j}
\end{array}\right] \leq k_{1} \hat{e}
$$

which can be viewed as a collision-avoidance formulation between two robots, where $I_{2} \in \mathbb{R}^{2 \times 2}$ denotes an identity matrix. Expanding to $N$ WMRs, let $\dot{Z}_{r_{i}}=\dot{Z}_{\mathrm{cmd}_{i}}-k_{2}\left(Z_{\mathrm{ref}_{i}}-\right.$ $\left.Z_{\mathrm{cmd}_{i}}\right)$ and

$$
\begin{aligned}
\dot{Z}_{\mathrm{ref}} & =\left[\begin{array}{c}
\dot{Z}_{\mathrm{ref}_{1}} \\
\dot{Z}_{\mathrm{ref}_{2}} \\
\vdots \\
\dot{Z}_{\mathrm{ref}_{N}}
\end{array}\right] \in \mathbb{R}^{2 N}, \quad \dot{Z}_{r}=\left[\begin{array}{c}
\dot{Z}_{r_{1}} \\
\dot{Z}_{r_{2}} \\
\vdots \\
\dot{Z}_{r_{N}}
\end{array}\right] \in \mathbb{R}^{2 N} \\
u & =\left[\begin{array}{c}
u_{1} \\
u_{2} \\
\vdots \\
u_{N}
\end{array}\right] \in \mathbb{R}^{2 N}, \quad A=\left[\begin{array}{cccc}
A_{1} & 0 & \cdots & 0 \\
0 & A_{2} & \cdots & 0 \\
\vdots & \vdots & \ddots & 0 \\
0 & 0 & \cdots & A_{N}
\end{array}\right] \in \mathbb{R}^{2 N \times 2 N}
\end{aligned}
$$

we can obtain

$$
\begin{array}{cl}
\min _{u} & \left\|\dot{Z}_{\mathrm{ref}}-\dot{Z}_{r}\right\|^{2} \\
\text { s.t. } & u^{-} \leq u \leq u^{+} \\
& -2 H C A u \leq B_{\text {right }}
\end{array}
$$

in which

$$
u^{-}=\left[\begin{array}{c}
u_{1}^{-} \\
u_{2}^{-} \\
\vdots \\
u_{N}^{-}
\end{array}\right] \in \mathbb{R}^{2 N}, \quad u^{+}=\left[\begin{array}{c}
u_{1}^{+} \\
u_{2}^{+} \\
\vdots \\
u_{N}^{+}
\end{array}\right] \in \mathbb{R}^{2 N}
$$

the matrix $H=\operatorname{blkdiag}(M) \in \mathbb{R}^{\left(N^{2}-N\right) / 2 \times\left(N^{2}-N\right)}$ with $M=\left[m_{12}, m_{13}, \ldots, m_{1 N}, m_{23}, \ldots, m_{i j}, \ldots, m_{(N-1) N}\right] \in$ $\mathbb{R}^{1 \times\left(N^{2}-N\right)}$, blkdiag $(\bullet)$ denotes a diagonal matrix, and $m_{i j}$

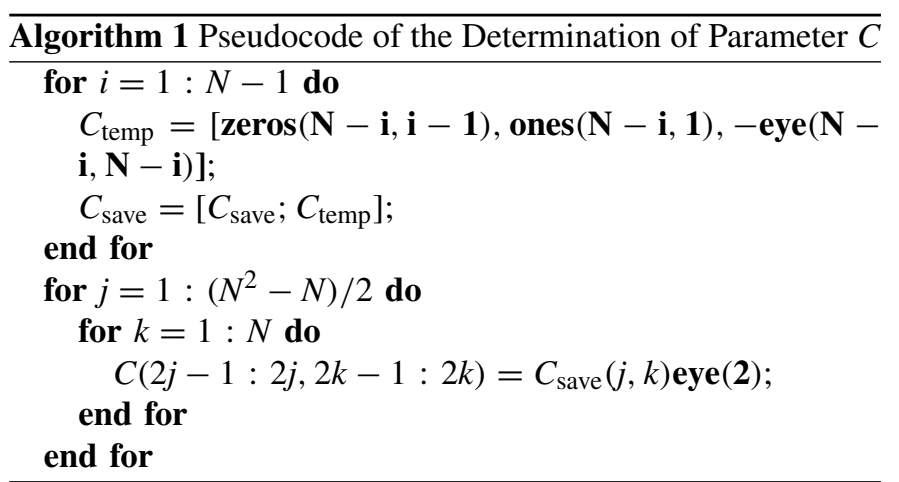

is defined as $m_{i j}=\left(Z_{\text {ref }_{i}}-Z_{\text {ref }_{j}}\right)^{\mathrm{T}}, i=1, \ldots, N-1$ and $j=i+1, \ldots, N . B_{\text {right }}=k_{1}\left(\xi-d^{2}\right)$ and

$$
\xi=\left[\begin{array}{c}
\left\|Z_{\mathrm{ref}_{1}}-Z_{\mathrm{ref}_{2}}\right\|^{2} \\
\left\|Z_{\mathrm{ref}_{1}}-Z_{\mathrm{ref}_{3}}\right\|^{2} \\
\vdots \\
\left\|Z_{\mathrm{ref}_{N-1}}-Z_{\mathrm{ref}_{N}}\right\|^{2}
\end{array}\right] \in \mathbb{R}^{\left(N^{2}-N\right) / 2}
$$

for $C, C \in \mathbb{R}^{\left(N^{2}-N\right) \times 2 N}$, which is composed of 0 , 1 , and -1 . For clarity, we give pseudocode of the determination of parameter $C$, as shown in Algorithm 1, where zeros $(\bullet)$, ones( $(\bullet)$, and $\operatorname{eye}(\bullet)$ denote the zero matrix, one matrix, and identity matrix, respectively. Letting $B=-2 H C A$, the kinematic control problem of $N$ WMRs integrating collision-avoidance, trajectory tracking, and velocity bound constraints is summarized as

$$
\begin{array}{cl}
\min _{u} & \left\|\dot{Z}_{\text {ref }}-\dot{Z}_{r}\right\|^{2} \\
\text { s.t. } & u^{-} \leq u \leq u^{+} \\
& B u \leq B_{\text {right }} .
\end{array}
$$

Remark 2: In real applications, every WMR is equipped with several sensors to sensors surrounding environmental information around itself. Therefore, when other WMRs enter the detection range of a WMR, the position information around the neighboring WMRs is easier to obtain. Therefore, in essence, the designed MWMRs' kinematic control scheme with certified safety can be viewed as a distributed framework since the robots do not need to communicate with each other. To make the design process of the following controller clear, we give (17) in a fashion of combination.

\section{B. Controller Design}

To find the minimal solution of the resultant constrained optimization problem (17), we design a Lagrangian-based controller to recursively solve (17). Specifically, a Lagrange function is first defined

$$
L(u \in \Omega, \lambda)=\left\|\dot{Z}_{\text {ref }}-\dot{Z}_{r}\right\|^{2}+\lambda^{\mathrm{T}}\left(B u-B_{\text {right }}\right)
$$

where $\lambda \in \mathbb{R}^{\left(N^{2}-N\right) / 2}$ is the Lagrange multiplier. $\Omega$ is a set, which is chosen to be $\Omega=\left\{u \in \mathbb{R}^{2 N}, u^{-} \leq u \leq u^{+}\right\}$to bound the wheel velocities of the robots. According to the 


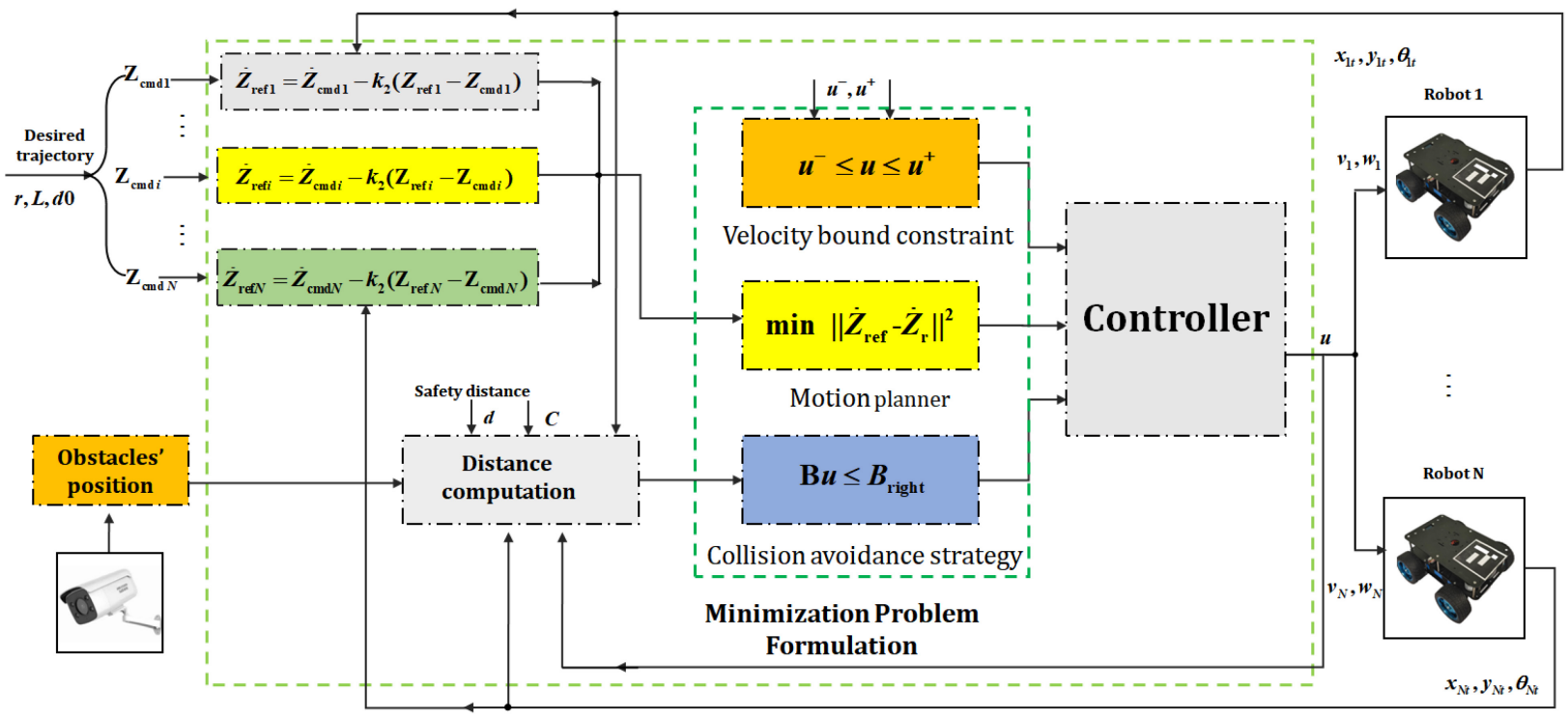

Fig. 3. Control block diagram of the developed controller (23) for solving optimization problem (17).

KKT conditions, an optimal solution of (17) can be written as the following equations:

$$
\begin{aligned}
& u=P_{\Omega}\left(u-\frac{\partial L}{\partial u}\right) \\
& \begin{cases}\lambda=0, & \text { if } B u \leq B_{\text {right }} \\
\lambda>0, & \text { otherwise }\end{cases}
\end{aligned}
$$

in which $P_{\Omega}: \mathbb{R}^{2 N} \rightarrow \Omega=\left\{u \in \mathbb{R}^{2 N}, u^{-} \leq u \leq u^{+}\right\}$ is a piecewise-linear projection operation to a set $\Omega$. Based on [44]-[46], $P_{\Omega}(u)=\left[\left[P_{\Omega}(u)\right]_{1},\left[P_{\Omega}(u)\right]_{2}, \ldots,\left[P_{\Omega}(u)\right]_{2 N}\right]$ with its $i$ th element defined as

$$
\left[P_{\Omega}(u)\right]_{i}= \begin{cases}u_{i}^{-}, & \text {if } u_{i} \leq u_{i}^{-} \\ u_{i}, & \text { if } u_{i}^{-} \leq u_{i} \leq u_{i}^{+} \\ u_{i}^{+}, & \text {if } u_{i} \geq u_{i}^{+} .\end{cases}
$$

Equation (20) can be further written as

$$
\lambda=\max \left(\left(\lambda+B u-B_{\text {right }}\right), 0\right) .
$$

Overall, the designed Lagrangian-based controller is

$$
\begin{aligned}
& \epsilon \dot{u}=-u+P_{\Omega}\left(u-2 A^{\mathrm{T}}\left(A u-\dot{Z}_{r}\right)-B^{\mathrm{T}} \lambda\right) \\
& \epsilon \dot{\lambda}=\max \left(\left(B u-B_{\text {right }}+\lambda\right), 0\right)-\lambda
\end{aligned}
$$

where $\epsilon>0$ is a constant that is associated with the convergence speed of the controller (23). Based on our experience feedback from experiments, $\epsilon \in(0,0.1)$ can deliver the better performance. In addition, $\lambda=0$ always holds for $Z_{\mathrm{ref}_{i}} Z_{\mathrm{ref}_{i}} \geq d$, which means other robots, or say obstacles, do not threaten the safety of robot $i$. Only when the collision criterion is satisfied $Z_{\text {ref }_{i}} Z_{\text {ref }_{i}}<d$, (23b) comes into force, and $\lambda>0$ due to the collision-avoidance behavior. Algorithm 2 presents the pseudocode of the designed controller (23) for the simultaneous trajectory tracking and collision-avoidance of MWMRs. In addition, a topological graph of the controller (23) for solving (17) is given in Fig. 3 to form a better illustration and enhance readability. Fig. 4 presents a block diagram for the configuration of the controller.

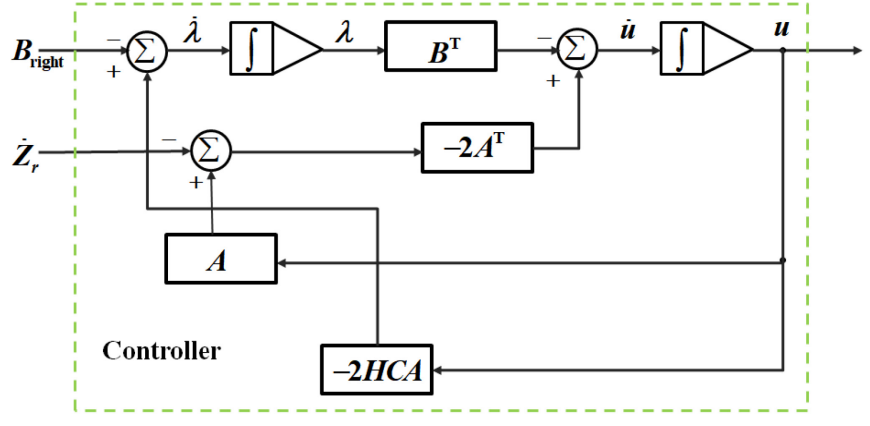

Fig. 4. Block diagram for the configuration of the controller.

Remark 3: To achieve simultaneous collision-avoidance and target tracking, together with velocity limits, of a group of WMRs, we formulate these problems as an optimization problem. The Lagrange multiplier method is employed in the controller to obtain the optimal solution. Zhang et al. [47]-[49] proposed a power-type varying-parameter control method. To be brief, the solving procedure was divided into three steps: first, $\partial L(u, \lambda) / \partial u$ and $\partial L(u, \lambda) / \partial \lambda$ were rewritten as a linear matrix equation formatted by $W Y-G=0$. Through finding zeros of the linear matrix equation by constructing the error function evolution formulation $\dot{e}=-k e$ with $e=W Y-G$ and $k=\gamma+t^{\gamma}$, an optimal solution of the optimization problem was found. The method was proven to provide a faster convergence speed and inherent noise tolerance compared to the traditional zeroing method. Mapping to our study, let

$$
W=\left[\begin{array}{ccc}
2 A^{\mathrm{T}} A & B^{\mathrm{T}} & 0 \\
B & 0 & l \\
0 & 2 \tilde{l} & \tilde{\lambda}
\end{array}\right], \quad Y=\left[\begin{array}{c}
u \\
\lambda \\
l
\end{array}\right], \quad G=\left[\begin{array}{c}
2 A^{\mathrm{T}} \dot{Z}_{r} \\
B_{\text {right }} \\
0
\end{array}\right]
$$

and we will obtain a linear matrix equation corresponding to the resultant optimization problem (17). In this regard, it is obvious that the difference between the method used in this article and the power-type varying-parameter control method 


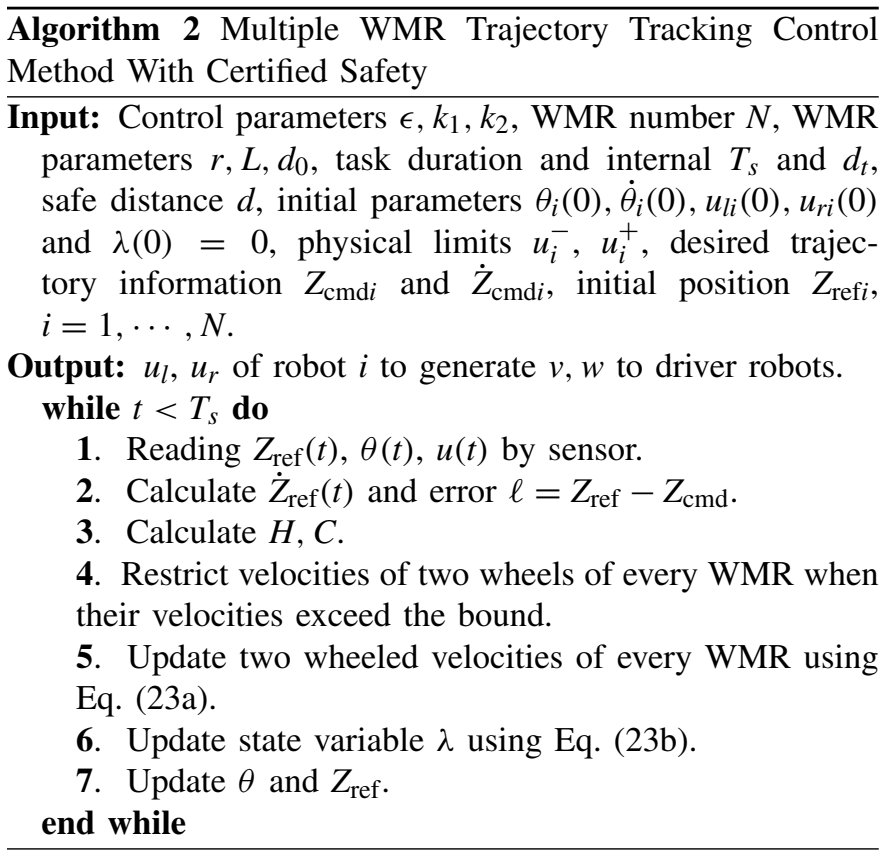

lies in the updation of control variable $u$ and state variable $\lambda$. In addition to these two algorithms, for instance, six numerical algorithms proposed in [46] are also a feasible scheme. In the current study, we focus on extending an optimization-based inequality obstacle avoidance method to mobile robots and achieving simultaneous obstacle avoidance and target tracking for a group of robots. The majority of the study consists of investigating the effectiveness of the optimization-based control scheme in MWMRs and ignoring the comparison between optimization algorithms. Note that as it would the investigation scope of this study, parameters involved in (24) are not introduced. Their corresponding explanations can be found in [48].

\section{Theoretical Analyses}

In this section, the convergence of the designed controller is given. The resultant collision-avoidance inequality is shown to eliminate collisions between MWMRs.

Definition 1 [50]: A function $\mathcal{H}(\bullet)$ is said to be monotone if it is continuously differentiable and ensures that $\nabla \mathcal{H}+(\nabla \mathcal{H})^{\mathrm{T}}$, where $\nabla \mathcal{H}$ is the gradient of $\mathcal{H}(\bullet)$, is positive semidefinite.

Lemma 1 [45], [50]: Let $S$ be a compact set that is positively invariant with respect to the dynamics of a system $\dot{x}=f(x)$. Let $V=V(x) \in \mathbb{R}$ be a continuously differentiable function, such that $\dot{V} \leq 0$ in $S$. Let $E$ be the set of all points in $S$. Let $E$ be the set of all points in $S$, where $\dot{V}=0$. Let $M$ be the largest invariant set in $E$. Thus, every solution starting in $S$ approaches $M$ as $t \rightarrow \infty$.

Lemma 2 [45], [50]: If the designed controller satisfies

$$
\kappa \dot{\boldsymbol{x}}=-\boldsymbol{x}+\boldsymbol{P}_{S}(\boldsymbol{x}-\varrho \mathcal{H}(\boldsymbol{x}))
$$

it will globally converge to the optimal solution of the solved problem, where $\kappa>0$ and $\varrho>0$ are constant parameters, and the definition $\boldsymbol{P}_{S}$ is the same as $P_{\Omega}$.

Theorem 1: The designed controller (23) globally converges to the optimal solution of (18). Under its control, tracking error to the desired trajectory converges to 0 , and collisionavoidance is achieved.

Proof: The proof is composed of three steps, that is: 1) convergence of the tracking error $Z_{\mathrm{ref}}-Z_{\mathrm{cmd}}$;2) global convergence of the constructed controller; and 3) effectiveness of the proposed collision-avoidance strategy.

Step 1: Define the tracking error as $\ell=Z_{\text {ref }}-Z_{\mathrm{cmd}}$, where $Z_{\text {ref }} \in \mathbb{R}^{2 N}$ represents the reference point sets, and $Z_{\text {cmd }} \in \mathbb{R}^{2 N}$ represent the desired trajectory sets corresponding to $N$ WMRs. Now, we construct a Lyapunov function as $V=\ell^{\mathrm{T}} \ell / 2$. Obviously, $V=\ell^{\mathrm{T}} \ell / 2 \geq 0$ and $V=0$ only when $\ell=0$.

Computing the time derivation of $V$, we can obtain $\dot{V}=$ $\ell^{\mathrm{T}} \dot{\ell}=-k_{2} \ell^{\mathrm{T}} \ell \leq 0$ and $\dot{V}=0$ only if $\ell=0$. Based on Lemma 1 , the tracking error globally converges to 0 when $t \rightarrow \infty$

Step 2: The above-designed RNN controller (23) can further be rewritten as

$$
\epsilon\left[\begin{array}{c}
\dot{u} \\
\dot{\lambda}
\end{array}\right]=\left[\begin{array}{c}
-u+P_{\Omega}\left(u-2 A^{\mathrm{T}}\left(A u-\dot{Z}_{r}\right)-B^{\mathrm{T}} \lambda\right) \\
-\lambda+\max \left(\left(B u-B_{\text {right }}+\lambda\right), 0\right)
\end{array}\right] .
$$

Let $\chi=[u, \lambda]^{\mathrm{T}}$. Equation (23) is reformulated as

$$
\epsilon \chi=-\chi+P_{\bar{\Omega}}(\chi-\mathcal{H}(\chi))
$$

where

$$
\mathcal{H}(\chi)=\left[\begin{array}{c}
2 A^{\mathrm{T}}\left(A u-\dot{Z}_{r}\right)+B^{\mathrm{T}} \lambda \\
-B u+B_{\text {right }}
\end{array}\right]
$$

For (29), we can obtain

$$
\nabla \mathcal{H}=\partial \mathcal{H} / \partial \chi=\left[\begin{array}{cc}
2 A^{\mathrm{T}} A & B^{\mathrm{T}} \\
-B & 0
\end{array}\right] .
$$

$\nabla \mathcal{H}(\chi)+(\nabla \mathcal{H})^{\mathrm{T}}(\chi)=\left[\begin{array}{cc}4 A^{\mathrm{T}} A & 0 \\ 0 & 0\end{array}\right]$, based on Definition 1, and $\nabla \mathcal{H}(\chi)+\nabla \mathcal{H}^{\mathrm{T}}(\chi)$ is positive semidefinite. Therefore, $\mathcal{H}(\chi)$ is a monotone function. Following (27), it satisfies Lemma 1 , where $\kappa=\epsilon$ and $\varrho=1 . P_{S}=P_{\bar{\Omega}}=\left[P_{\Omega} ; P_{\Lambda}\right]$, in which $P_{\Omega}$ is the same as the definition of (19), and $P_{\Lambda}=\max (\bullet, 0)$ is a special projection operator to $\mathbb{R}$. Following Lemma 2, it can be concluded that the designed RNN controller (23) globally converges to the optimal solution of (17).

Step 3: Define $D=\left\|Z_{\text {ref }_{i}} Z_{\text {ref }_{j}}\right\|-d$. Inequality (7) is rewritten as

$$
\dot{D} \geq-k_{1} D
$$

and $\dot{D}=0$ only if $D=0$. We further rewrite $D$ as $D \geq e^{-k_{1} t} D(0)$, where $D(0)$ denotes the initial value of $D$. Obviously, $D \geq 0$, and $D=0$ only if $D(0)=0$. Since the control gain parameter $k_{1}$ is a positive constant, $\dot{D} \leq 0 . \dot{D}=0$ is only if $D(0)=0$. Therefore, we can easily obtain that $\left\|Z_{\text {ref }_{i}} Z_{\text {ref }_{j}}\right\| \geq d$ when $\dot{D} \geq-k_{1} D$.

Remark 4: Note that $D(0)=\left\|Z_{\text {ref }_{i}}(0) Z_{\text {ref }_{j}}(0)\right\|-d \geq 0$ is assumed in the above analyses. In general, the WMR almost never collides with the environmental obstacle or other WMRs at the initial time in the actual scene. 


\section{Simulation}

Simulations are conducted in this part to substantiate the effectiveness of the control scheme (23). Without a loss of generality, the point obstacle was first investigated, where five cases: 1) collision-avoidance between a single WMR with multiple static environmental obstacles; 2) collision-avoidance between a single WMR and dynamic environmental obstacles; 3) collision-avoidance between MWMRs without considering environmental obstacles; 4) collision-avoidance between MWMRs considering multiple static environmental obstacles; and 5) collision-avoidance between MWMRs considering both static and dynamic environmental obstacles, were considered. We then considered obstacles with general significance. Based on the bounding box idea, environmental obstacles were enveloped with several rectangles of different sizes. Finally, we considered a scene with more WMRs to show further applications of the proposed MWMR control scheme.

\section{A. Experiments Setup}

The following simulations were performed on a notebook computer equipped with an Intel Core $2.20-\mathrm{GHz}$ i5-5200U CPU, 12-GB memory, and Windows 10, 64-bit operating system. The controller parameter $\epsilon$ was valued as 0.02 , and control gain parameters were $k_{1}=k_{2}=8$. In addition, the experimental internal was set as $d_{t}=0.005$ s. $T_{s}$ was determined by the task performed by the robot. The safe distance $d$ was set as $d=0.3 \mathrm{~m}$ (in Fig. $10, d=0.5$ $\mathrm{m})$. Initial attitude $\theta_{i}(0), \dot{\theta}_{i}(0)$, and initial wheel velocities $u_{l i}(0)$ and $u_{r i}(0)$ corresponding to every WMR were set to 0 , $i=1,2, \ldots, N$. Here, parameters $k_{1}, k_{2}$, and $\epsilon \in(0,0.1)$ are usually adjusted manually based on the experimental requirement. They determine the obstacle avoidance behavior, the tracking accuracy of the robot with respect to the desired trajectory, and the convergence rate of the designed controller, respectively. Theoretically, the larger $k_{1}\left(k_{2}\right)$ is, the larger the intensity of obstacle avoidance (the better the tracking accuracy with respect to the desired trajectory), but at the expense of increasing computational complexity. Moreover, if the obstacle avoidance intensity is too high, the robot will have a large instantaneous acceleration during obstacle avoidance, which is not expected nor considered a positive outcome.

Remark 5: Among simulation, three groups of WMR parameters are used: one is $r=0.4 \mathrm{~m}, L=1.85 \mathrm{~m}$ with $u_{i}^{-}=-u_{i}^{+}=-2 \mathrm{~m} / \mathrm{s}$, and $d_{0}=0.75 \mathrm{~m}$ (corresponding to Figs. 5 and 6$)$. The other is $r=1.5 \mathrm{~m}, L=3 \mathrm{~m}$ with $u_{i}^{-}=-u_{i}^{+}=-4 \mathrm{~m} / \mathrm{s}$, and $d_{0}=L / 2$ (corresponding to Figs. 7-9). Parameters used in Fig. 10 are $r=0.04 \mathrm{~m}$, $L=0.185 \mathrm{~m}$, and $d_{0}=0.075 \mathrm{~m}$, which are consistent with the WMR parameters used in the physical experiment. This is to show feasibility of our scheme applied to various types of WMRs.

\section{B. Point Obstacle Collision-Avoidance}

1) Single Robot Multiple Static Obstacle CollisionAvoidance: In this experiment, the robot was expected to move 4-m forward along a straight line from $[0,0]^{\mathrm{T}}$ at a speed of $0.1 \mathrm{~m} / \mathrm{s}$. We set up two static obstacles, $O_{1}$ and $O_{2}$, whose (a)

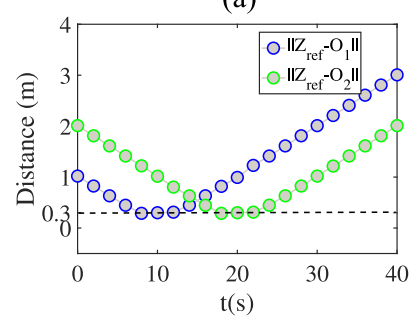

(c)

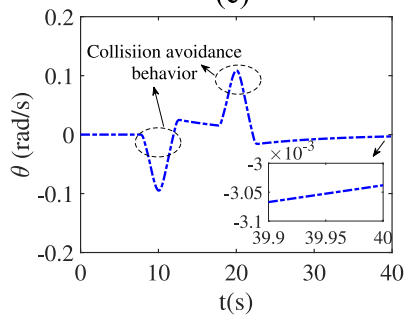

(e)

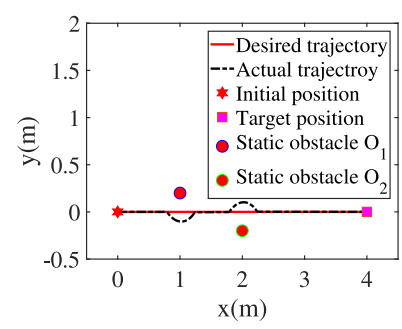

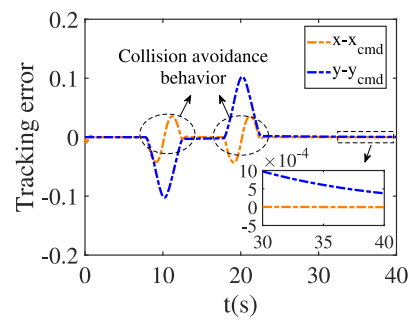

(b)

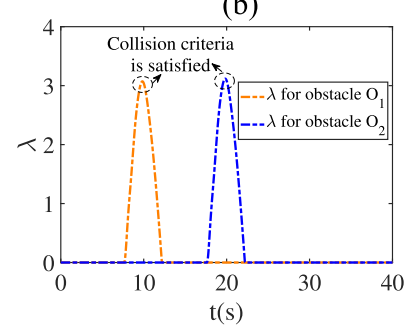

(d)

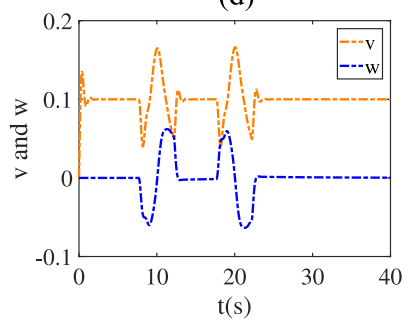

(f)
Fig. 5. Collision avoidance and the desired trajectory tracking results between a single robot and multiple environmental obstacles, where two static obstacles are considered. (a) Tracking result. (b) Tracking error $\left\|Z_{\text {ref }}-Z_{\mathrm{cmd}}\right\|$ at the $x$-axis and $y$-axis. (c) Distance profiles $\left\|Z_{\text {ref }}-O_{1}\right\|$ and $\left\|Z_{\text {ref }}-O_{2}\right\|$. (d) State variables $\lambda$. (e) Heading angle $\theta$ profile. (f) Profiles of linear velocity $v$ and angular velocity $w$ of the robot.

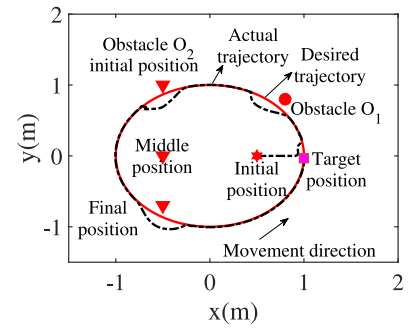

(a)

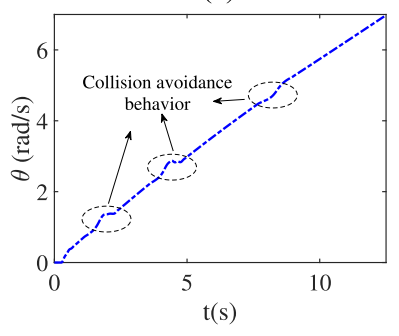

(c)

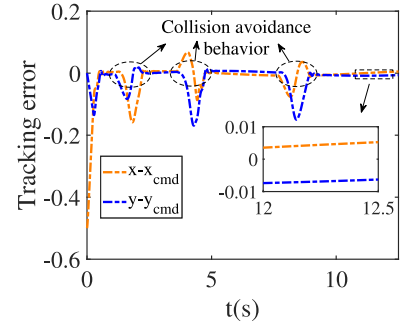

(b)

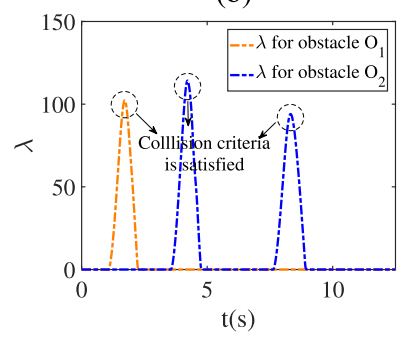

(d)
Fig. 6. Collision avoidance and the desired trajectory tracking results between a single robot and a dynamic obstacle (triangle). (a) Tracking result. (b) Tracking error $\left\|Z_{\text {ref }}-Z_{\text {cmd }}\right\|$ at the $x$-axis and $y$-axis. (c) Heading angle $\theta$ profile. (d) State variables $\lambda$.

positions are $[1,0.2]^{\mathrm{T}}$ and $[2,0.2]^{\mathrm{T}}$, to show the collisionavoidance behavior of the robot. For intuitive observation, the initial position of the robot was set to $[0,0]^{\mathrm{T}}$. Simulation 

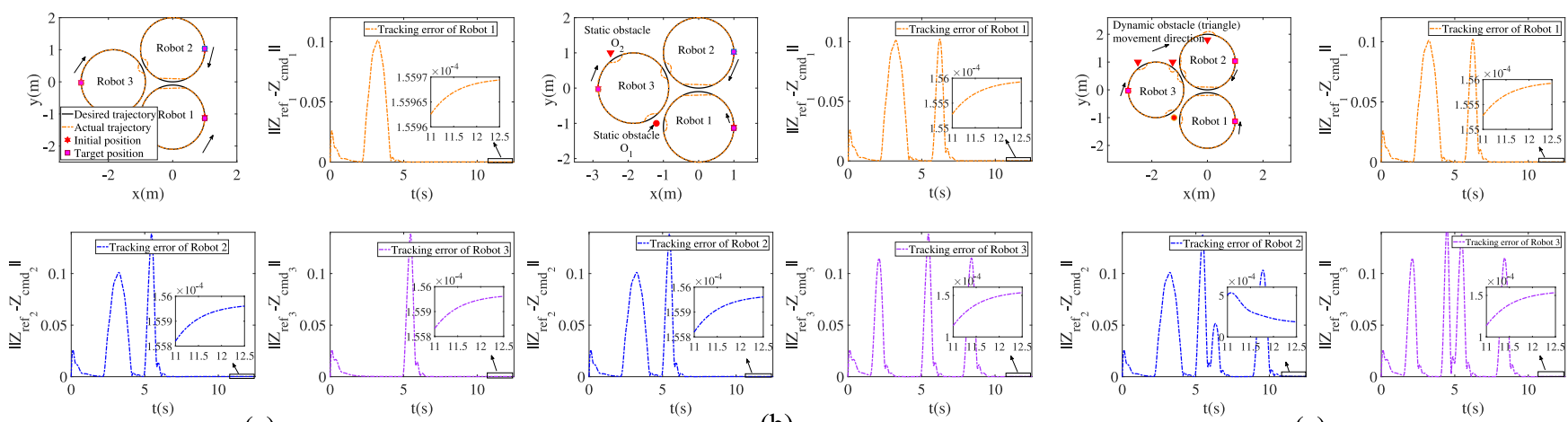

(b)

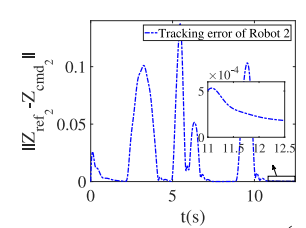

(c)

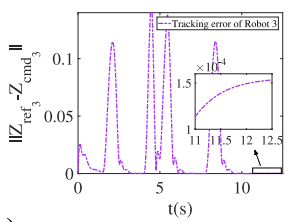

)

Fig. 7. Collision avoidance based on different environments. (a) Collision avoidance between the MWMRs without the environmental obstacle. (b) MWMR collision-avoidance considering the environmental static obstacle. (c) MWMR collision-avoidance considering the environmental dynamic obstacle. From top to down and from left to right are the tracking results and the tracking error corresponding to the WMRs 1,2 , and 3 , respectively.

results achieved by the controller (23) are shown in Fig. 5. Fig. 5(a) illustrates the collision-avoidance result and trajectory tracking result, where the desired trajectory is denoted by a red line. The black line denotes the trajectory achieved by the robot. The hexagon denotes the initial position of the WMR, and the square denotes the target position. Fig. 5(a) and (c) shows that the WMR successfully avoided the collision with two static obstacles and always kept a safe distance that is greater than 0.3 from two obstacles $O_{1}$ and $O_{2}$. Outside the nonsafety region (i.e., $\left\|Z_{\text {ref }}-O_{i}\right\| \geq d, i=1,2$ ) means that the obstacles $O_{1}$ and $O_{2}$ did not threaten the safety of the WMR, and the WMR moved along the desired trajectory because the trajectory tracking task was prominent and the collision-avoidance was not activated. The tracking errors $\left\|Z_{\text {ref }}-Z_{\text {cmd }}\right\|$ at both the $x$-axis and the $y$-axis were at the $10^{-4}$ level at $t=[30,40] \mathrm{s}$ [see Fig. 5(b)]. Around $t=10 \mathrm{~s}$ and $t=20 \mathrm{~s}$, the distances between robot and obstacles $O_{1}$ and $\mathrm{O}_{2}$ satisfied the collision criteria. The collision-avoidance strategy (17c) came into force, where $\lambda>0$ [see Fig. 5(d)]. At this time, the top priority in the control task was collisionavoidance. The heading angle $\theta$ of the robot changed because the robot steered to avoid obstacles $O_{1}$ and $O_{2}$ [see Fig. 5(e)]. Due to the collision-avoidance behavior, the tracking error of the robot was greater than 0. Fig. 5(f) shows the linear velocity $v$ profile and the angular velocity $w$ profile of the robot. Outside the nonsafety region, the robot moved at the desired linear velocity of $0.1 \mathrm{~m} / \mathrm{s}$. To avoid obstacles $O_{1}$ and $O_{2}$, the robot steered so that $v$ and $w$ changed. Here, profiles of the left wheel's velocity $u_{l}$ and the right wheel's velocity $u_{r}$ are not given since they can be easily derived based on both Fig. 5(f) and (3).

2) Single Robot Dynamic Obstacles Collision-Avoidance: In practice, an obstacle is not always static. We considered the collision-avoidance problem between the WMR and the dynamic obstacle. The desired trajectory was a circle; the tracking trajectory was defined as $[0+\cos (0.5 t), 0+$ $\sin (0.5 t)]^{\mathrm{T}}$. The task duration was set as $T_{s}=12.5 \mathrm{~s}$. The dynamic obstacle $\mathrm{O}_{2}$ was denoted by a triangle, whose position varied three times along the movement direction. Circle obstacle $O_{1}$ was still considered to be static. In this experiment, the initial position of the robot was set to $[0.5,0]^{\mathrm{T}}$. Fig. 6 shows the corresponding simulation result. Fig. 6(a) shows that the WMR achieved collision-avoidance three times during its movement. For the middle position of the dynamic obstacle, the collision-avoidance was not activated because the collision criteria were not satisfied. Fig. 6(b)-(d) shows the tracking error profiles, the heading angle $\theta$ profile, and the state variable $\lambda$ profile, respectively. At one point, the heading angle of the WMR was 0 . This is because the robot located at $[0.5,0]^{\mathrm{T}}$ needed to approach the initial position $[1,0]^{\mathrm{T}}$ of the desired trajectory. Based on Fig. 6, the conclusion is similar to that obtained from Experiment 1. If the collision-avoidance strategy was not activated, then $\lambda=0$. The top priority in the control task was the trajectory tracking task. If not, the collision-avoidance task was prominent, which resulted in changes in $\lambda$ and $\theta$ and in tracking error.

3) Collision-Avoidance Between the MWMRs: The existing obstacle avoidance schemes mainly focus on the collisionavoidance between the applied object and the environmental obstacles. In this experiment, we cast light on the collisionavoidance between MWMRs. The number of the WMR was chosen to be 3, where WMR 1 moved anti-clockwise and the other two WMRs moved clockwise from different specified initial positions so that they encountered each other. The desired trajectory tracking results of the three robots and obstacle avoidance results are shown in Fig. 7(a). From top to bottom and from left to right are the tracking results and the tracking error corresponding to WMRs 1,2 , and 3, respectively. At $t=2 \mathrm{~s}$, WMRs 1 and 2 encountered each other. Since the distance between them met the collision criterion, the collision-avoidance strategy assumed control, and their respective tracking errors reached 0.1. By enabling the distance between WMRs 1 and WMR 2 to be maintained at a setting safety threshold of 0.3 , safety was ensured. WMRs 2 and WMR 3 encountered each other at $t=5 \mathrm{~s}$. Under the designed obstacle collision strategy, as expected, they also successfully escaped each other and maintained a safe distance. For all WMRs, after their distance was outside the safe distance, they returned to their predefined paths. Under the designed controller, the tracking errors of three robots converged to zero outside the nonsafety region.

4) MWMR Collision-Avoidance Considering Static Environmental Obstacles: In real applications, a WMR should not only escape the neighboring WMRs but also avoid 

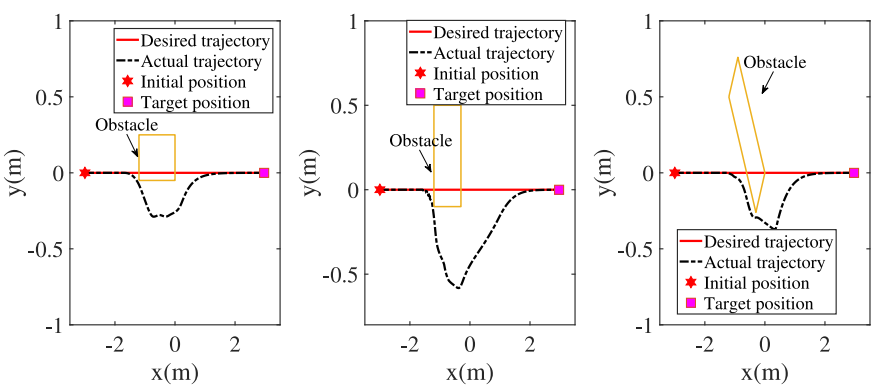

Fig. 8. Experimental results corresponding to the single enveloping shape environmental obstacle, where the obstacle was enveloped as a rectangle with different attitudes based on the encountered obstacle shape.

encountered environmental obstacles. Two static environmental obstacles were considered. Experimental results are illustrated in Fig. 7(b). For WMRs 1 and 3, while avoiding neighboring robots, they also avoided collisions with the encountered environmental obstacles. When WMR 3 was close to the triangle and circle obstacles, its movement direction deviated from the desired trajectory to maintain a safe distance from the encountered environmental obstacles. After keeping a safe distance from the obstacle, WMR 3 returned to the desired path step by step and moved along the desired path, with a promising tracking error of $10^{-4}$, until it reached the destination. We also observed that the tracking error of WMR 3 changed three times since the collision-avoidance strategy mechanism participated in the control process three times.

5) MWMR Collision-Avoidance Considering Dynamic Environmental Obstacles: Fig. 7(c) shows the simulation results corresponding to a case that considers MWMR collision-avoidance as well as collision-avoidance with static and dynamic obstacles. Similar to the previous illustrated simulation results, outside the nonsafety region, the three robots moved along the desired trajectory with a promising tracking error that converged to 0 . Inside the nonsafety region, the collision-avoidance strategy was activated by keeping a safe distance from the environmental obstacles and other WMRs, such that safety was ensured.

\section{Generalized Obstacle Collision-Avoidance}

We considered obstacles with general significance. Based on the bounding box idea, they can be enveloped as a geometric shape, such as a circle, a rectangle, or a combination of them, denoted by a set of points. By describing the distance between the WMR and the enveloping obstacle as a point-to-point distance, the collision-avoidance between the WMR and the complicated environmental obstacle was easy to achieve. Fig. 8 shows the experimental results corresponding to a single environmental obstacle enveloped as a rectangle. Obstacle avoidance in a complicated environment is shown in Fig. 9, where the obstacle is assumed to be enveloped as a combination of several geometric shapes, as shown in cyan. Fig. 9(a) and (b) shows U-shaped and right-angle-shaped trajectory tracking results corresponding to one WMR and two WMRs, respectively. Following them, we observed that all WMRs moved along the respective expected trajectories. Only

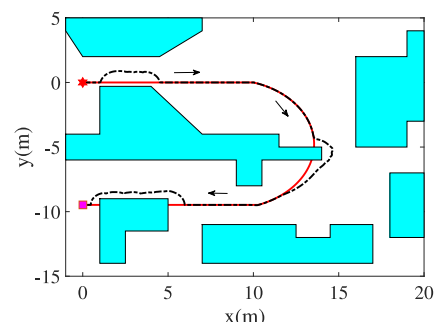

(a)

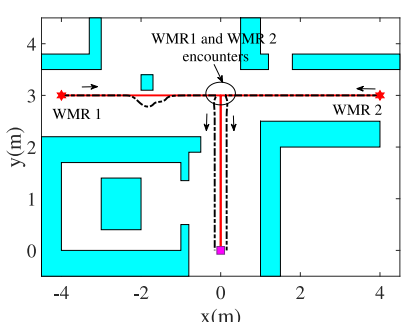

(b)
Fig. 9. Obstacle avoidance in a complicated environment. The obstacle was assumed to be enveloped as a combination of several geometric shapes, as shown in cyan. (a) U-shaped trajectory tracking corresponding to one WMR. (b) Right-angle-shaped trajectory tracking corresponding to two WMRs.

when the obstacle was detected, and their distance satisfied the collision criterion, did the WMRs deviate from the respective expected trajectory to avoid collisions. After keeping a safe distance that was greater than or equal to $d$, the WMRs returned to the desired trajectory. In addition to avoiding the environmental obstacles, WMRs 1 and 2 encountered each other at the mark shown in Fig. 9(b). Since their trajectories were the same, they each followed a trajectory parallel to the desired trajectory after $4 \mathrm{~s}$.

\section{Extension}

In the previous experiments, the number of WMRs was chosen as 3 or 1 to show the feasibility of the control scheme (23) for the simultaneous target tracking and obstacle avoidance of MWMRs. A common scene with more WMRs was considered to show further applications of the control scheme. As shown in Fig. 10(b), WMRs were required to reach a specific location along the desired trajectory. Because the WMRs may come from different directions, they will inevitably encounter each other during movement. We simplified every route into six lines, shown in different colors in Fig. 10(b), where the arrow denotes the movement direction of the WMR. Fig. 10(a) shows the eventual simulation result. Under the designed controller, six WMRs successfully escaped the other WMRs encountered during their movement. When obstacles did not threaten the safety of robots, they moved along their respective trajectories. Fig. 10(c)-(i) provides snapshots of the experimental results corresponding to different times. Before $t=10 \mathrm{~s}$, because all WMRs were far away from each other, the motion planner played a leading role in the controller and had all WMRs perform trajectory tracking tasks. It can be seen from Fig. 10(c) that, as expected, six robots successfully tracked their respective straight-line trajectories under the designed controller. At $t=10-13 \mathrm{~s}$, the distance between WMR 1 and WMR 2 satisfied the collision criterion (as did WMR 3 and WMR 6), and the collision-avoidance strategy was activated. At this time, the top priority of the control task was collision-avoidance instead of the trajectory tracking task. Therefore, WMR 1 and WMR 2, together with WMR 3 and WMR 6, steered and deviated from their respective trajectories [see Fig. 10(d)]. Benefitting from $\lambda>0$, the distance between WMR 1 and WMR 2, and the distance between WMR 3 and WMR 6, maintained a safety threshold of $0.5 \mathrm{~m}$ during collision-avoidance. 


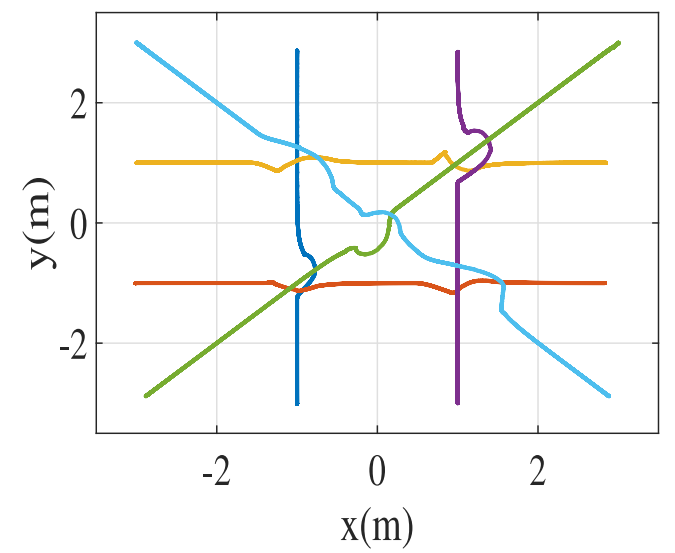

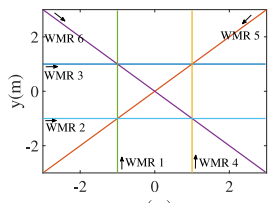

(b)

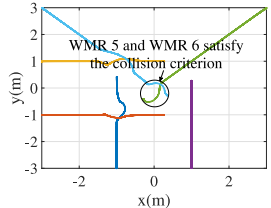

(f)

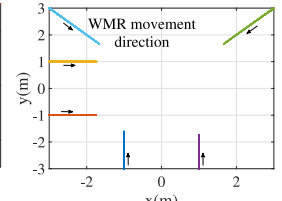

(c)

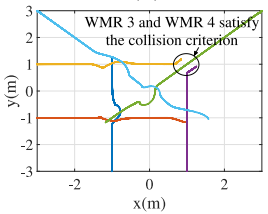

(g)

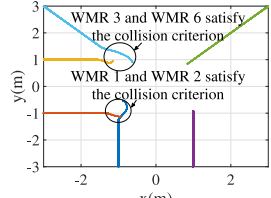

(d)

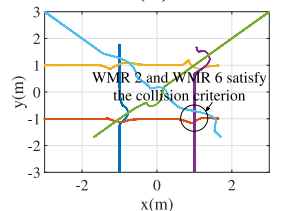

(h)

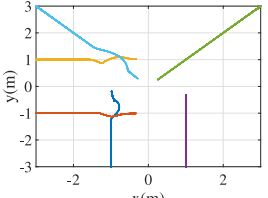

(e)

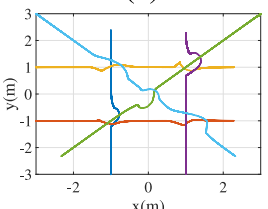

(i)

(a)

Fig. 10. Snapshot of six WMRs' collision-avoidance results at different times, where the safe distance is set as $0.5 \mathrm{~m}$. (a) Tracking result at $t=30 \mathrm{~s}$. (b) Desired trajectories corresponding to six WMRs. (c) $t=10 \mathrm{~s}$. (d) $t=13 \mathrm{~s}$. (e) $t=15 \mathrm{~s}$. (f) $t=17 \mathrm{~s}$. (g) $t=20 \mathrm{~s}$. (h) $t=22 \mathrm{~s}$. (i) $t=25 \mathrm{~s}$.

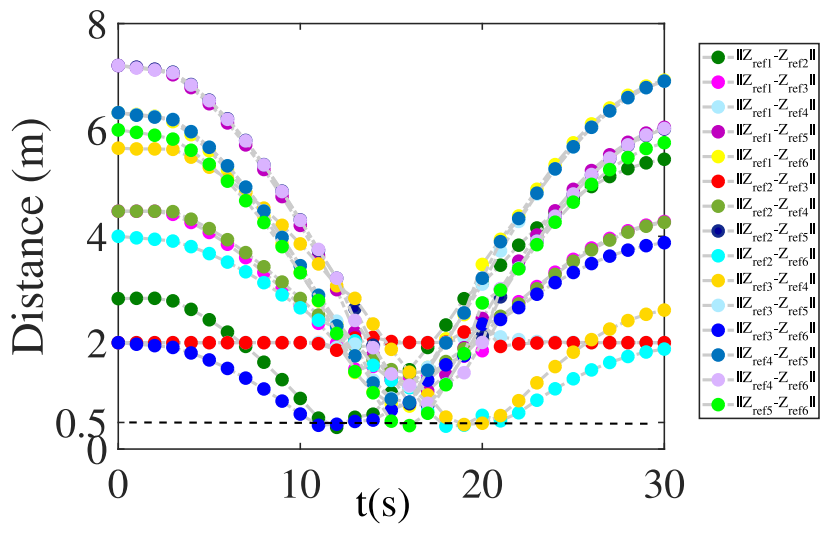

Fig. 11. Distance profiles corresponding to Fig. 10, which shows that distances between all robots were greater than or equal to the safety threshold $0.5 \mathrm{~m}$ under the proposed controller. Based on the setting collision-avoidance criterion, no collision is shown in Fig. 10.

For WMR 4 and WMR 5, it was determined by distance computation that they had no risk of collision at the present time. The top priority in the control tasks of both WMR 4 and WMR 5 was still trajectory tracking. Therefore, the collisionavoidance strategy was not activated with $\lambda=0$. However, when $t=17 \mathrm{~s}$ [see Fig. 10(d)], WMR 6 threatened the safety of WMR 5. To keep a safe distance from WMR 6, the collision-avoidance strategy of WMR 5 was activated. At this time, for WMR 1 and WMR 2, and for WMR 3 and 6, since they had no risk of collision, $\lambda$ decreasing to 0 , and the motion planner was again dominant. Similarly, when $t=20 \mathrm{~s}$ and $t=22 \mathrm{~s}$, the collision criterion was satisfied for WMR 1 and WMR 4 and for WMR 2 and WMR 6, respectively. They also successfully avoided their respective encountered robots as expected. After $t=25 \mathrm{~s}$ [see Fig. 10(i)], no robot encountered any other robots again. Therefore, all robots moved along the desired straight-line trajectory. At $t=30 \mathrm{~s}$, all WMRs stopped. Fig. 11 shows the distance profiles corresponding to Fig. 10. It can be seen that distances between robots were greater than or equal to the safety threshold of $0.5 \mathrm{~m}$, based on the collision-avoidance criterion, which shows that no collisions occurred.

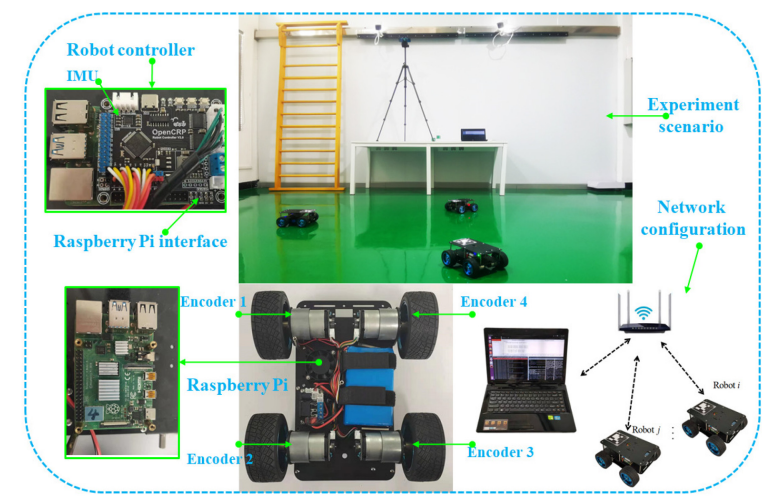

Fig. 12. Experimental scenario and configuration.

\section{Physical Experiments}

To show the feasibility of the proposed control scheme in real applications, a physical experiment was implemented in Xtark mobile robots with four differentially driven wheels. The basic parameters of the Xtark robot are listed in Table II. In this experiment, $d_{0}$ was valued as $7.5 \mathrm{~cm}$ to choose the mass center of the robot as the reference point. The experimental scenario and configuration are shown in Fig. 12. The WMR and notebook computer were connected to a common WIFI network by a router. The notebook computer was set as the master, and it controlled the movement of the robots by a robot operating system (ROS). A mobile phone placed on a tripod was used to photograph the motion state of the MWMRs while they executed the desired task. The controller used in the WMR was an opensource control module for the ROS on Pi (OpenCRP), which can be embedded directly on Raspberry Pies.

Figs. 13-15 of the three physical experiments correspond to one WMR's collision-avoidance; two WMRs' collisionavoidance, where a WMR can be viewed as a dynamic obstacle for another WMR; and three WMRs' collision-avoidance, respectively. The mark on the robot indicates the forward direction of the robot. As shown in Fig. 13, the WMR needs to track a U-shaped trajectory. Two mineral water bottles 


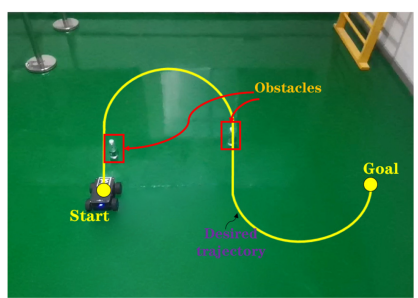

(a)

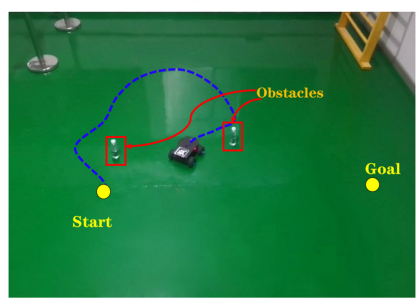

(e)

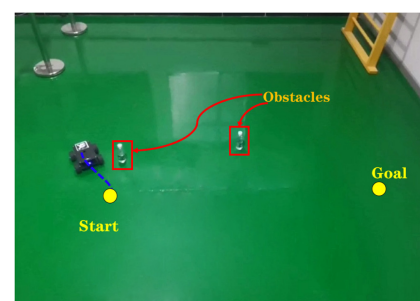

(b)

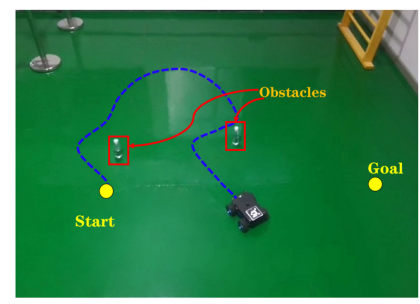

(f)

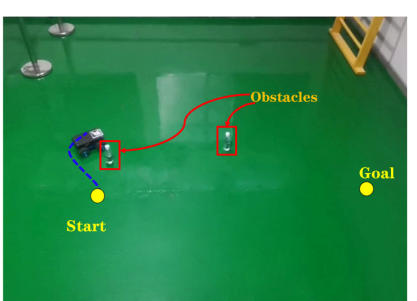

(c)

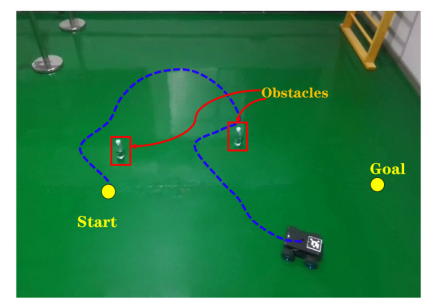

(g)

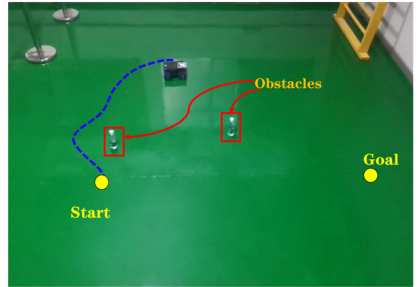

(d)

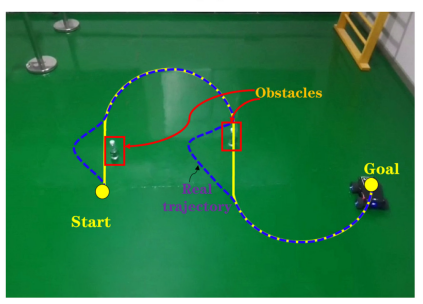

(h)

Fig. 13. Environmental obstacles collision-avoidance illustration: snapshot of single WMR collision-avoidance for a U-shaped path where two static obstacles are placed on the path. (a) Desired trajectory. (b) and (e) Collision avoidance behavior. (c) and (f) WMR returns to its desired trajectory when obstacles do not threaten the safety of the WMR. (d) and (g) WMR moves along the circle trajectory, at which point the trajectory tracking task is the top priority. (h) Real trajectory.

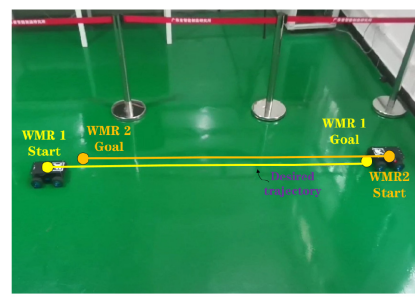

(a)

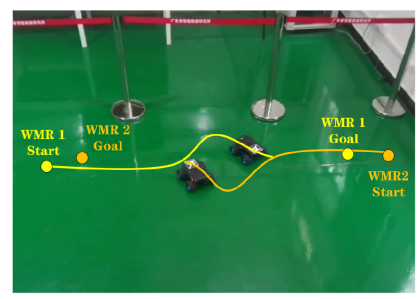

(c)

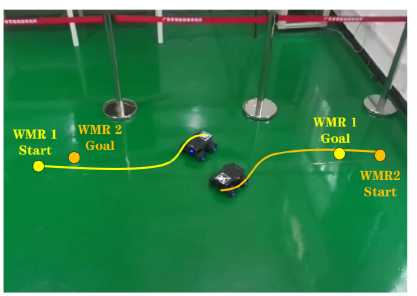

(b)

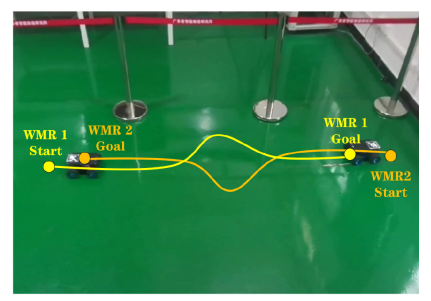

(d)
Fig. 14. Dynamic obstacles collision-avoidance illustration: snapshot of two WMRs' collision-avoidance, where they would encounter each other at the halfway point of the desired tracking path. (a) Desired trajectory. (b) Collision avoidance behavior. (c) Robots return to their respective desired trajectories. (d) Real trajectories achieved by robots.

were placed on the path, as shown in Fig. 13(a), to show the collision-avoidance behavior of the WMR. Fig. 13(b) and (e) shows the WMR avoiding mineral water bottles. Since the distance between the WMR and bottles was detected to be less than the safety threshold, collision-avoidance was activated so that the WMR steers. When the mineral water bottle did not threaten the safety of the WMR, the WMR would gradually return to the desired path since the trajectory tracking became a top priority again, as shown in Fig. 13(c) and (f). Unlike the robot's behavior when avoiding the first mineral water bottle, the time taken for the robot to return to the desired trajectory was longer than the former when the WMR avoided the second bottle, and there was some shaking, albeit for a short period of time. This phenomenon can be observed in the attached
TABLE II

Basic Parameters of the Used Xtark Robot

\begin{tabular}{ccc}
\hline & Length & $23.2 \mathrm{~cm}$ \\
Xtark & Width & $14.0 \mathrm{~cm}$ \\
& Height & $9.5 \mathrm{~cm}$ \\
& Weight & $2.5 \mathrm{~kg}$ \\
\hline \multirow{3}{*}{ Wheel } & Diameter & $8 \mathrm{~cm}$ \\
& Thickness & $3.3 \mathrm{~cm}$ \\
& Wheelbase & $18.5 \mathrm{~cm}$ \\
\hline
\end{tabular}

video. Fig. 13(d) and (g) are snapshots showing the WMR tracking the circle trajectory. When $\lambda=0$, the WMR moved along the desired trajectory, and the WMR stopped when it reached its destination. Fig. 14 is a snapshot corresponding to the two WMRs, where two robots that are $2.5 \mathrm{~m}$ away from each other gradually approach each other with an equal forward velocity of $0.1 \mathrm{~m} / \mathrm{s}$. Fig. 14(a) and (d) shows the initial state and the final state of the two WMRs, respectively. Fig. 14(b) and (c) indicates the collision-avoidance behavior of the WMRs. Fig. 14 shows that, in the beginning, the two robots moved along the desired straight-line trajectory. When the two robots were close to each other, the position distance norm between them satisfied the collision criteria. The two robots deviated from the desired trajectory to satisfy the safety rule [see Fig. 14(b)]. As shown in Fig. 14(c), the two robots again steered to return to the desired trajectory. When no control signal was sent to the robots, the WMR stopped. The three WMRs' collision-avoidance is shown in Fig. 15(a). As expected, every WMR successfully avoided collision with the other two WMRs and reached their respective destinations. The dynamic obstacle avoidance process corresponding to the above physical experiments is shown in the attached video.

Remark 6: Among the simulation and physical experiments, multiple groups of wheel parameters $(r$ and $L)$ were given and tested to show the feasibility of our method applied to 


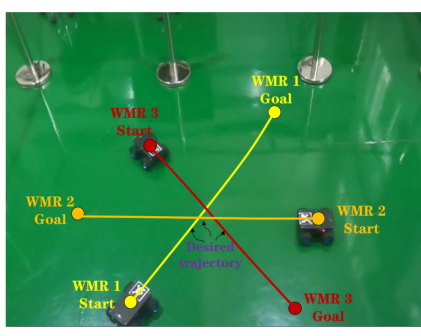

(a)

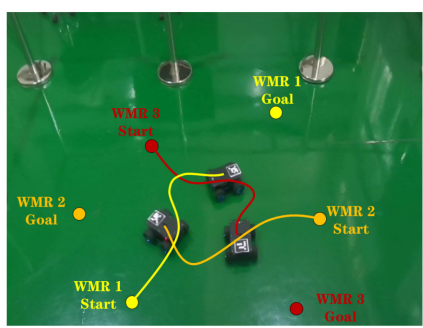

(c)

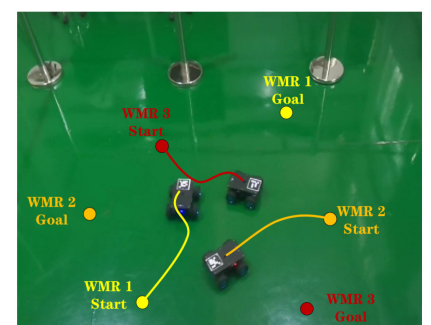

(b)

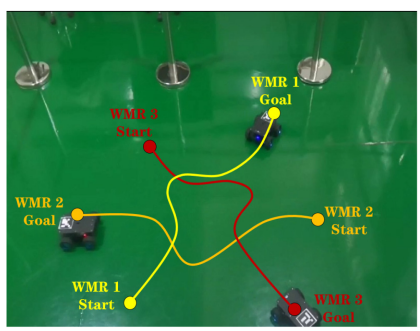

(d)
Fig. 15. Multiple robots' collision-avoidance illustration: snapshot of three WMRs' collision-avoidance. (a) Desired trajectory. (b) Collision avoidance behavior. (c) Robots return to their respective desired trajectories when the other two robots do not threaten the safety of a robot. (d) Real trajectories.

differentially driven WMRs with different sizes. Based on the simulation results, the path achieved by the WMR is smooth and stable. However, it is necessary to point out that in our experiments, the real trajectory of the robot was different from the simulation results to some extent. This may be due to the road condition, wheels, car size and shape, or communication response delay. This phenomenon could be weakened by appropriately increasing the safe distance or adjusting control parameters.

\section{CONCLUSION}

A Lagrangian-based dynamic controller was designed for the simultaneous obstacle avoidance and target tracking of MWMRs. Three objects were achieved, successfully incorporating the path following both collision-avoidance and robot velocity compliance. Theoretical analyses showed that the controller globally converges to the optimal solution of the problem. Numerical experiments and physical experiments substantiated the feasibility of the controller and theoretical analyses. If obstacles do not threaten the safety of the robot, the top priority in the controller is the target tracking task and $\lambda=0$. All robots move along their respective desired trajectories with the desired tracking error. If the distance between a robot and environmental obstacle or other robots satisfies the collision criterion, the collision-avoidance strategy is activated and leads the controller, enabling the WMR to always keep a safe distance $\geq d$ from other objects by steering. A case with more WMRs has also been considered, showing the potential application of the proposed scheme.

In a future study, we will be devoted to simultaneous cooperative kinematic control and collision-avoidance control of MWMRs under partially known information in unknown environments. How to weaken the influence of feedback gain parameters $k_{1}, k_{2}$, and $\epsilon$ on the system performance is also of interest.

\section{REFERENCES}

[1] Z. Zhang, H. Xu, Z. Chao, X. Li, and C. Wang, "A novel vehicle reversing speed control based on obstacle detection and sparse representation,” IEEE Trans. Intell. Transp. Syst., vol. 16, no. 3, pp. 1321-1334, Jun. 2015.

[2] J. Li, G. Deng, C. Luo, Q. Lin, Q. Yan, and Z. Ming, "A hybrid path planning method in unmanned air/ground vehicle (uav/ugv) cooperative systems," IEEE Trans. Veh. Technol., vol. 65, no. 12, pp. 9585-9596, Dec. 2016.

[3] C. Guindel, D. Martin, and J. M. Armingol, "Fast joint object detection and viewpoint estimation for traffic scene understanding," IEEE Intell. Transp. Syst. Mag., vol. 10, no. 4, pp. 74-86, Dec. 2018.

[4] C. Yang, G. Peng, Y. Li, R. Cui, C. Long, and Z. Li, "Neural networks enhanced adaptive admittance control of optimized robot-environment interaction," IEEE Trans. Cybern., vol. 49, no. 7, pp. 2568-2579, Jul. 2019.

[5] L. Xiang, Y. Pan, C. Gong, and H. Yu, "Adaptive human-Robot interaction control for robots driven by series elastic actuators," IEEE Trans. Robot., vol. 33, no. 1, pp. 169-182, Feb. 2017.

[6] A. V. Savkin and H. Teimoori, "Decentralized navigation of groups of wheeled mobile robots with limited communication," IEEE Trans. Robot., vol. 26, no. 6, pp. 1099-1104, Dec. 2010.

[7] B.-S. Choi, J.-W. Lee, J.-J. Lee, and K.-T. Park, "A hierarchical algorithm for indoor mobile robot localization using RFID sensor fusion," IEEE Trans. Ind. Electron., vol. 58, no. 6, pp. 2226-2235, Jun. 2011.

[8] Y. Toda and N. Kubota, "Self-localization based on multiresolution map for remote control of multiple mobile robots," IEEE Trans. Ind. Informat., vol. 9, no. 3, pp. 1772-1781, Aug. 2013.

[9] M. A. H. Ali and M. Mailah, "Path planning and control of mobile robot in road environments using sensor fusion and active force control," IEEE Trans. Veh. Technol., vol. 68, no. 3, pp. 2176-2195, Mar. 2019.

[10] C. Yang, Z. Li, and L. Jing, "Trajectory planning and optimized adaptive control for a class of wheeled inverted pendulum vehicle models," IEEE Trans. Cybern., vol. 43, no. 1, pp. 24-36, Feb. 2013.

[11] J. Liao, Z. Chen, and B. Yao, "Model-based coordinated control of fourwheel independently driven skid steer mobile robot with wheel-ground interaction and wheel dynamics," IEEE Trans. Ind. Informat., vol. 15, no. 3, pp. 1742-1752, Mar. 2019.

[12] S. Li, R. Kong, and Y. Guo, "Cooperative distributed source seeking by multiple robots: Algorithms and experiments," IEEE/ASME Trans. Mechatron., vol. 19, no. 6, pp. 1810-1820, Dec. 2014.

[13] G. Hu, "Robust consensus tracking of a class of second-order multiagent dynamic systems," Syst. Control Lett., vol. 61, no. 1, pp. 134-142, 2012.

[14] B. Li, J. Chang, and C. Wu, "A potential function and artificial neural network for path planning in dynamic environments based on self-reconfigurable mobile robot system," in Proc. IEEE Int. Symp. Safety Security Rescue Robot. (SSRR), 2012, pp. 1-6, doi: $10.1109 /$ SSRR.2012.6523900.

[15] Y. Huang et al., "A motion planning and tracking framework for autonomous vehicles based on artificial potential field elaborated resistance network approach," IEEE Trans. Ind. Electron., vol. 67, no. 2, pp. 1376-1386, Feb. 2020.

[16] A. H. Khan, S. Li, and X. Luo, "Obstacle avoidance and tracking control of redundant robotic manipulator: An RNN-based metaheuristic approach," IEEE Trans. Ind. Informat., vol. 16, no. 7, pp. 4670-4680, Jul. 2020.

[17] H. Qu, S. X. Yang, A. R. Willms, and Z. Yi, "Real-time robot path planning based on a modified pulse-coupled neural network model," IEEE Trans. Neural Netw., vol. 20, no. 11, pp. 1724-1739, Nov. 2009.

[18] K.-T. Song and J.-Y. Lin, "Behavior fusion of robot navigation using a fuzzy neural network," in Proc. IEEE Int. Conf. Syst., vol. 6, 2006, p. $4910-4915$.

[19] R. Kala, A. Shukla, R. Tiwari, S. Rungta, and R. R. Janghel, "Mobile robot navigation control in moving obstacle environment using genetic algorithm, artificial neural networks and a* algorithm," in Proc. Wri World Congr. Comput. Sci. Inf. Eng. (CSIE), 2009, pp. 705-713, doi: 10.1109/CSIE.2009.854.

[20] J. E. Meng and D. Chang, "Obstacle avoidance of a mobile robot using hybrid learning approach," IEEE Trans. Ind. Electron., vol. 52, no. 3, pp. 898-905, Jun. 2005. 
[21] M. J. Phinni, A. P. Sudheer, and M. RamaKrishna, "Obstacle avoidance of a wheeled mobile robot: A genetic-neuro-fuzzy approach," in Proc. Int. Conf. Adv. Mech. Eng. (ICAME), 2008, pp. 1-3.

[22] L. Ren, W. Wang, and Z. Du, "A new fuzzy intelligent obstacle avoidance control strategy for wheeled mobile robot," in Proc. Int. Conf. Mechatron. Autom. (ICMA), 2012, pp. 1732-1737, doi: 10.1109/ICMA.2012.6284398.

[23] C.-J. Kim and D. Chwa, "Obstacle avoidance method for wheeled mobile robots using interval type-2 fuzzy neural network," IEEE Trans. Fuzzy Syst., vol. 23, no. 3, pp. 677-687, Jun. 2015.

[24] T. Dierks and S. Jagannathan, "Neural network control of mobile robot formations using rise feedback," in IEEE Trans. Syst., Man, Cybern. B, Cybern., vol. 39, no. 2, pp. 332-347, Apr. 2009.

[25] Y. Wang, D. Wang, S. Yang, and M. Shan, "A practical leader-follower tracking control scheme for multiple nonholonomic mobile robots in unknown obstacle environments," IEEE Trans. Control Syst. Technol., vol. 27, no. 4, pp. 1685-1693, Jul. 2019.

[26] Z. Xu, X. Zhou, and S. Li, "Deep recurrent neural networks based obstacle avoidance control for redundant manipulators," Frontiers Neurorobotics, vol. 13, no. 47, Jul. 2019

[27] D. Guo and Y. Zhang, "Acceleration-level inequality-based MAN scheme for obstacle avoidance of redundant robot manipulators," IEEE Trans. Ind. Electron., vol. 61, no. 12, pp. 6903-6914, Dec. 2014.

[28] Y. Zhang and J. Wang, "Obstacle avoidance for kinematically redundant manipulators using a dual neural network," IEEE Trans. Syst., Man, Cybern. B, Cybern., vol. 34, no. 1, pp. 752-759, Feb. 2004

[29] D. Guo and Y. Zhang, "A new inequality-based obstacle-avoidance MVN scheme and its application to redundant robot manipulators," IEEE Trans. Syst., Man, Cybern. C, Appl. Rev., vol. 42, no. 6, pp. 1326-1340, Nov. 2012.

[30] S. Li, J. He, Y. Li, and M. U. Rafique, "Distributed recurrent neural networks for cooperative control of manipulators: A game-theoretic perspective," IEEE Trans. Neural Netw. Learn. Syst., vol. 28, no. 2, pp. 415-426, Feb. 2017.

[31] X. Li, Z. Xu, S. Li, H. Wu, and X. Zhou, "Cooperative kinematic control for multiple redundant manipulators under partially known information using recurrent neural network," IEEE Access, vol. 8, pp. 40029-40038, 2020.

[32] L. Jin, S. Li, B. Hu, and C. Yi, "Dynamic neural networks aided distributed cooperative control of manipulators capable of different performance indices," Neurocomputing, vol. 291, pp. 50-58, May 2018.

[33] Z. Xu, S. Li, X. Zhou, S. Zhou, T. Cheng, and Y. Guan, "Dynamic neural networks for motion-force control of redundant manipulators: An optimization perspective," IEEE Trans. Ind. Electron., vol. 68, no. 2, pp. 1525-1536, Feb. 2021.

[34] Y. Zhang, S. Li, S. Kadry, and B. Liao, "Recurrent neural network for kinematic control of redundant manipulators with periodic input disturbance and physical constraints," IEEE Trans. Cybern., vol. 49, no. 12, pp. 4194-4205, Dec. 2019.

[35] L. Xiao and Y. Zhang, "Acceleration-level repetitive motion planning and its experimental verification on a six-link planar robot manipulator," IEEE Trans. Control Syst. Technol., vol. 21, no. 3, pp. 906-914, May 2013.

[36] D. Chen, S. Li, and L. Liao, "A recurrent neural network applied to optimal motion control of mobile robots with physical constraints," Appl. Soft Comput., vol. 85, pp. 1568-1580, Dec. 2019.

[37] Z. Zhang, S. Chen, J. Xie, and S. Yang, "Two hybrid multiobjective motion planning schemes synthesized by recurrent neural networks for wheeled mobile robot manipulators," IEEE Trans. Syst., Man, Cybern., Syst., early access, Jun. 17, 2020, doi: 10.1109/TSMC.2019.2920778.

[38] Z. Zhang, L. Zheng, Z. Chen, L. Kong, and H. R. Karimi, "Mutualcollision-avoidance scheme synthesized by neural networks for dual redundant robot manipulators executing cooperative tasks," IEEE Trans. Neural Netw. Learn. Syst., vol. 32, no. 3, pp. 1052-1066, Mar. 2021.

[39] T. Wang, Y. Wu, J. Liang, C. Han, J. Chen, and Q. Zhao, "Analysis and experimental kinematics of a skid-steering wheeled robot based on a laser scanner sensor," Sensors, vol. 15, no. 5, pp. 9681-9702, 2015.

[40] R. Fierro and F. L. Lewis, "Control of a nonholonomic mobile robot using neural networks," IEEE Trans. Neural Netw., vol. 9, no. 4, pp. 589-600, Jul. 1998

[41] V. Gazi and B. Fidan, "Coordination and control of multi-agent dynamic systems: Models and approaches," in Proc. Int. Workshop Swarm Robot., 2006, pp. 71-102.

[42] T. Xu, Y. Guan, J. Liu, and X. Wu, "Image-based visual servoing of helical microswimmers for planar path following," IEEE Trans. Autom. Sci. Eng., vol. 17, no. 1, pp. 325-333, Jan. 2020.
[43] X. Wu, J. Liu, C. Huang, M. Su, and T. Xu, "3-D path following of helical microswimmers with an adaptive orientation compensation model," IEEE Trans. Autom. Sci. Eng., vol. 17, no. 2, pp. 823-832, Apr. 2020.

[44] Y. Zhang, J. Li, M. Mao, W. Li, and S. Fu, "Complete theory for e47 and 94lvi algorithms solving inequality-and-bound constrained quadratic program efficiently," in Proc. Chin. Autom. Congr. (CAC), 2016, pp. 183-189, doi: 10.1109/CAC.2015.7382493.

[45] S. Li, Y. Zhang, and L. Jin, "Kinematic control of redundant manipulators using neural networks," IEEE Trans. Neural Netw. Learn. Syst., vol. 28 , no. 10 , pp. 2243-2254, Oct. 2017.

[46] Z. Zhang et al., "Comparisons among six numerical methods for solving repetitive motion planning of redundant robot manipulators," in Proc. IEEE Int. Conf. Robot. Biomimet. (ROBIO), 2018, pp. 1645-1652, doi: 10.1109/ROBIO.2018.8665072.

[47] Z. Zhang et al., "Robustness analysis of a power-type varying-parameter recurrent neural network for solving time-varying QM and QP problems and applications," IEEE Trans. Syst., Man, Cybern., Syst., vol. 50, no. 12, pp. 5106-5118, Dec. 2020.

[48] Z. Zhang, L. D. Kong, and L. Zheng, "Power-type varying-parameter RNN for solving TVQP problems: Design, analysis, and applications," IEEE Trans. Neural Netw. Learn. Syst., vol. 30, no. 8, pp. 2419-2433, Aug. 2019.

[49] Z. Zhang, X. Deng, L. Kong, and S. Li, "A circadian rhythms learning network for resisting cognitive periodic noises of time-varying dynamic system and applications to robots," IEEE Trans. Cognit. Develop. Syst., vol. 12 , no. 3 , pp. 575-587, Sep. 2020.

[50] L. Jin, S. Li, L. H. Manh, and X. Luo, "Manipulability optimization of redundant manipulators using dynamic neural networks," IEEE Trans. Ind. Electron., vol. 64, no. 6, pp. 4710-4720, Jun. 2017.

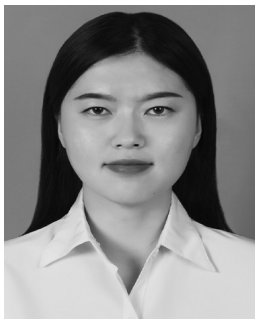

Xiaoxiao Li received the B.E. degree from Qufu Normal University, Shandong, China, in 2016, and the M.S. degree in computer science and technology from Qufu Normal University in 2019.

She currently works with the Robotics Team, Institute of Intelligent Manufacturing, Guangdong Academy of Sciences, Guangzhou, China. Her current research interests include intelligent optimization, neural networks, and robotic control.

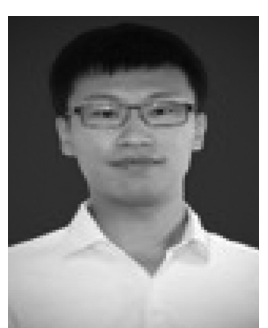

Zhihao Xu (Member, IEEE) received the B.E. and Ph.D. degrees from the School of Automation, Nanjing University of Science and Technology, Nanjing, China, in 2010 and 2016, respectively.

$\mathrm{He}$ is currently a Postdoctoral Fellow with the Robotics Team, Institute of Intelligent Manufacturing, Guangdong Academy of Sciences, Guangzhou, China. His main research interests include neural networks, force control, and intelligent information processing.

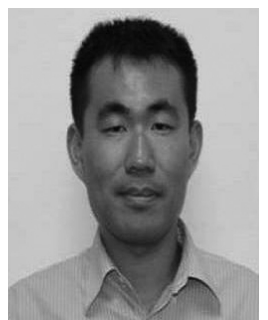

Shuai Li (Senior Member, IEEE) received the B.E. degree in precision mechanical engineering from the Hefei University of Technology, Hefei, China, in 2005, the M.E. degree in automatic control engineering from the University of Science and Technology of China, Hefei, in 2008, and the Ph.D. degree in electrical and computer engineering from the Stevens Institute of Technology, Hoboken, NJ, USA, in 2014

$\mathrm{He}$ is currently an Associate Professor (Reader) with the School of Engineering, Swansea University, Swansea, U.K., and also a Chief Scientist with the Technical Development Department, Foshan Tri-Co Intelligent Robot Technology Co., Ltd., Foshan, China. His current research interests include dynamic neural networks, robotic networks, and other dynamic problems defined on a graph. 


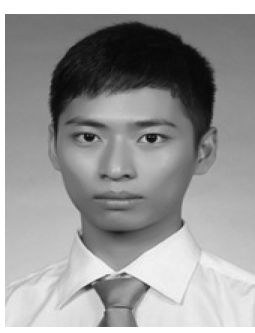

Zerong Su received the B.E. degree from the School of Electromechanical Engineering, Guangdong University of Technology, Guangzhou, China, in 2016.

$\mathrm{He}$ currently works with the Artificial Intelligence Research Center, Institute of Intelligent Manufacturing, Guangdong Academy of Sciences, Guangzhou. His research interests include SLAM using multisensor information fusion and path planning.

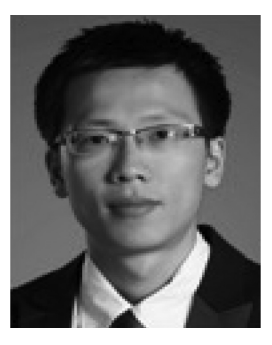

Xuefeng Zhou (Member, IEEE) received the M.E. and Ph.D. degrees in mechanical engineering from the South China University of Technology, Guangzhou, China, in 2006 and 2011, respectively.

$\mathrm{He}$ is currently the Director of the Robotics Team, Institute of Intelligent Manufacturing, Guangdong Academy of Sciences, Guangzhou. His research interests include neural networks, force control, and intelligent information processing.

Dr. Zhou won the Best Student Paper Award at the 2010 IEEE International Conference on Robotics and Biomimetics. 